\title{
First-Order Regularity of Convex Functions on Carnot Groups
}

\author{
By Matthieu Rickly
}

To the memory of $Q . G$.

\begin{abstract}
We prove that h-comvex functions on Carnot groups of step two are locally Lipschitz continuous with respect to any intrinsic metric. We show that an additional measurability condition implies the local Lipschitz continuity of h-convex functions on arbitrary Carnot groups.
\end{abstract}

\section{Introduction}

Recently, considerable efforts have been made in order to determine an appropriate notion of convexity in general Carnot groups and to establish regularity properties of convex sets and functions (see [5, 14, 2, 12, 13, 6, 16, 17, 11, 15]). In the sub-Riemannian setup of Carnot groups, the notion of geodetic convexity-which is perfectly appropriate in a Riemannian setting-has been shown to be inadequate in [17]. More promising notions of convexity in Carnot groups, $\mathrm{h}$ convexity and v-convexity, have been proposed in [5] and in [14]. Roughly, a function $u: \mathbb{G} \rightarrow \mathbb{R}$ defined on a Carnot group $\mathbb{G}$ is h-convex if it is convex along the integral curves of the left-invariant, horizontal vector fields. The aforementioned notions of convexity have turned out to be more or less equivalent: In the setting of Heisenberg groups, it has been shown that the classes of $\mathrm{h}$-convex and v-convex functions coincide [2]. In general Carnot groups the v-convex functions are precisely the upper semicontinuous $h$-convex functions (see $[21,18,15]$ ).

In the Euclidean setting, it is well known that convex functions are locally Lipschitz continuous. Moreover, due to a theorem of Aleksandrov, convex functions are twice differentiable almost everywhere (see, for instance, [8]). Proving similar regularity theorems for h-convex functions in a noncommutative Carnot group becomes a challenging problem which is a subject of current interest. Let us briefly recall the state of the art: The local Lipschitz continuity of h-convex functions on Heisenberg groups with respect to any intrinsic metric was proved in [2]. An intrinsic version of the Aleksandrov theorem for continuous, $h$-convex functions on Heisenberg groups has been obtained in [11] and [13]. This last result has been generalized to arbitrary Carnot groups of step two in [6]. One objective of the present article is to remove the continuity assumption in this second-order regularity result.

Math Subject Classifications. Primary 43A80; secondary 26B25.

Key Words and Phrases. Stratified groups, convex functions. 
Theorem 1.1. If $\mathbb{G}$ is a Carnot group of step two and $\Omega \subseteq \mathbb{G}$ is an $h$-convex, open subset, then every $h$-convex function $u: \Omega \rightarrow \mathbb{R}$ is locally Lipschitz continuous with respect to any intrinsic metric on $\mathbb{G}$.

Since an h-convex function $u: \Omega \rightarrow \mathbb{R}$ (where $\Omega$ is an h-convex, open subset of an arbitrary Carnot group $\mathbb{G}$ ) which is locally bounded above is also locally Lipschitz continuous, it turns out that the crucial point in the proof of Theorem 1.1 is to show that an h-convex function $u$ admits local upper bounds. The main geometric property of Carnot groups of step two used in the proof of the existence of these bounds is that any such group contains a finite set whose h-convex closure has nonempty interior. More formally, we say that a Carnot group $\mathbb{G}$ is finitely h-convex if there exists a finite subset $F \subseteq \mathbb{G}$ such that the smallest h-convex set $C \subseteq \mathbb{G}$ which contains $F$ has nonempty interior.

Theorem 1.2. Any Carnot group of step two is finitely h-convex.

The assumption on the step of the group in the preceding theorem cannot be relaxed. In Section 5, we exhibit a Carnot group of step 3 which is not finitely h-convex. Hence, our strategy to prove Theorem 1.1 breaks down in higher step.

Our approach in the general case is as follows: Let $\mathbb{G}$ denote a Carnot group of homogeneous dimension $Q$ and let $\mathcal{H}^{Q}$ denote $Q$-dimensional Hausdorff measure built with respect to an intrinsic metric (compare Section 2). We first study the topological boundary of measurable, h-convex subsets of $\mathbb{G}$ and we prove the following geometric/measure-theoretic result.

Theorem 1.3. There exists $0 \leq c<1$ such that

$$
\frac{\mathcal{H}^{Q}(B(g, r) \cap C)}{\mathcal{H}^{Q}(B(g, r))} \leq c
$$

for all $0<r<+\infty$, whenever $C \subseteq \mathbb{G}$ is an $h$-convex, measurable subset and $g \in \partial C$ is a point on its boundary.

We stress that the measurability assumption in Theorem 1.3 cannot be removed. Indeed, surprisingly, there exist nonmeasurable, h-convex sets in the first Heisenberg group $\mathbb{H}_{1}$ (cf. [18]). Theorem 1.3 has some interesting consequences. First, it can be used to give a concise alternative proof (cf. [18]) of the $L^{\infty}-L^{1}$ estimates for h-convex functions of Danielli, Garofalo, and Nhieu [5, Theorem 9.2]. Second, (1.1) can be combined with sufficient conditions proved by Danielli in [4] (see also [3]) to show that the boundary points of an h-convex, bounded open subset $\Omega$ of a Carnot group are regular and Hölder regular for weak solutions of the Dirichlet problem for the subelliptic $p$-Laplacian. The following continuity result is also a corollary of Theorem 1.3.

Theorem 1.4. Let $\mathbb{G}$ be a Carnot group, $\Omega \subseteq \mathbb{G}$ an h-convex, open subset and $u: \Omega \rightarrow \mathbb{R}$ a measurable, $h$-convex function. Then $u$ is locally Lipschitz continuous with respect to any intrinsic metric on $\mathbb{G}$.

As a direct consequence of Theorem 1.4, we obtain that if $\left\{u_{k}\right\}_{k \in \mathbb{N}}$ is a sequence of measurable h-convex functions $u_{k}: \Omega \rightarrow \mathbb{R}$ (where $\Omega$ is an h-convex, open subset of some Carnot group $\mathbb{G}$ ) that admits pointwise upper bounds, then $\lim \sup _{k \rightarrow \infty} u_{k}$ is again locally Lipschitz continuous.

We conclude this introductory section with an overview of the content of the article: In the second section, we recall the necessary background on Carnot groups and introduce the 
terminology and the notation. Moreover, we show that there is a rich supply of bounded hconvex sets with regular boundary in any Carnot group. More precisely, we show that there exists a countable basis of the topology consisting of bounded $h$-convex sets with smooth boundary (Corollary 2.6). In the third section, we show that h-convex functions which are locally bounded above are necessarily also locally bounded below and locally Lipschitz continuous with respect to any intrinsic metric. We prove Theorem 1.1 and Theorem 1.2 in Section 4. In Section 5, we exhibit a Carnot group of step three which is not finitely h-convex (Theorem 5.4). Section 6 is devoted to the proof of Theorem 1.3 and to the proof of a more general version of Theorem 1.4 (Theorem 6.5).

\section{Definitions and basic results}

\subsection{Carnot groups}

Definition 2.1. A connected, simply connected, nilpotent Lie group $\mathbb{G}$ is a Carnot group if its Lie algebra $\mathfrak{g}$ of left-invariant vector fields admits a stratification, i.e., if there exist nonzero subspaces $V_{1}, \ldots, V_{s}$ such that

(i) $\mathfrak{g}=\oplus_{i=1}^{s} V_{i}$,

(ii) $\left[V_{1}, V_{i}\right]=V_{i+1}(i=1, \ldots, s-1)$ and

(iii) $\left[V_{1}, V_{s}\right]=0$.

Given a stratification $\oplus_{i=1}^{s} V_{i}$ of $\mathfrak{g}$, we define $d_{i}=\operatorname{dim}_{\mathbb{R}}\left(V_{i}\right)(1 \leq i \leq s)$. The homogeneous dimension of $\mathbb{G}$ is $Q=\sum_{i=1}^{s} i d_{i}$.

Throughout the article, $\mathbb{G}$ denotes a Carnot group of topological dimension $d$ and step $s$, whose unit element is $e$, while $\mathfrak{g}$ denotes its Lie algebra of left-invariant vector fields, equipped with a fixed stratification $V_{1} \oplus \cdots \oplus V_{s}$.

The exponential mapping exp $: \mathfrak{g} \rightarrow \mathbb{G}$ is a global diffeomorphism and

$$
\exp (X) \cdot \exp (Y)=\exp (X * Y) \quad \forall X, Y \in \mathfrak{g} .
$$

Here $\cdot$ denotes the group operation in $\mathbb{G}$ and $X * Y$ is defined by the Baker-Campbell-DynkinHausdorff formula

$$
X * Y=X+Y+\frac{1}{2}[X, Y]+\frac{1}{12}[X,[X, Y]]+\frac{1}{12}[Y,[Y, X]]+\cdots,
$$

where the dots indicate a finite $\mathbb{R}$-linear combination of Lie brackets of $X$ and $Y$ of order $\geq 4$ (see, e.g., [19]).

For $g_{0} \in \mathbb{G}, l_{g_{0}}: \mathbb{G} \rightarrow \mathbb{G}$ and $r_{g_{0}}: \mathbb{G} \rightarrow \mathbb{G}$ denote, respectively, left and right translation by $g_{0}$, i.e.,

$$
l_{g_{0}}(g)=g_{0} \cdot g \quad \text { and } \quad r_{g_{0}}(g)=g \cdot g_{0} \quad \forall g \in \mathbb{G} .
$$

For each $\lambda>0$, let $A_{\lambda}: \mathfrak{g} \rightarrow \mathfrak{g}$ be the unique Lie algebra automorphism such that $A_{\lambda}(X)=\lambda^{i} X$ if $X \in V_{i}(i=1, \ldots, s)$. Then

$$
\delta_{\lambda}=\exp \circ A_{\lambda} \circ \exp ^{-1}
$$

is an automorphism of $\mathbb{G}$, and $\left\{\delta_{\lambda}\right\}_{\lambda>0}$ is a 1-parameter group of automorphisms of $\mathbb{G}$ called dilations. By convention, for all $g \in \mathbb{G}, \delta_{0}(g)=e$ and $\delta_{\lambda}(g)=\delta_{-\lambda}\left(g^{-1}\right)$ if $\lambda<0$. 
A left-invariant vector field $X$ on $\mathbb{G}$ is called horizontal if it belongs to the first layer $V_{1}$ of $\mathfrak{g}$. If $\left(X_{1}, \ldots, X_{d_{1}}\right)$ is a basis of $V_{1}$, then the subbundle $H \mathbb{G}$ of $\mathrm{T} \mathbb{G}$ spanned by $X_{1}(g), \ldots, X_{d_{1}}(g)$ at each $g \in \mathbb{G}$ is called the horizontal bundle. A Carnot group $\mathbb{G}$ can be equipped with an intrinsic sub-Riemannian metric in the following way: A curve $\gamma:[a, b] \rightarrow \mathbb{G}$ is said to be admissible if it is absolutely continuous and horizontal, i.e., if $\dot{\gamma}(t) \in \mathbf{H}_{\gamma(t)} \mathbb{G}$ for almost every $t \in[a, b]$. If $\langle\cdot, \cdot\rangle$ is a left-invariant inner product on the fibers of $\mathrm{HG}$, define the sub-Riemannian length of $\gamma$ to be

$$
L(\gamma)=\int_{a}^{b}\left(\langle\dot{\gamma}(t), \dot{\gamma}(t)\rangle_{\gamma(t)}\right)^{\frac{1}{2}} d t .
$$

The Carnot-Carathéodory distance $\rho\left(g_{1}, g_{2}\right)$ of points $g_{1}, g_{2} \in \mathbb{G}$-also called sub-Riemannian distance-is defined as the infimum of sub-Riemannian lengths of admissible curves connecting $g_{1}$ with $g_{2}$. It is well-known that $\rho$ is a left-invariant, homogeneous metric on $\mathbb{G}$, that is

$$
\rho\left(l_{g_{0}}\left(g_{1}\right), l_{g_{0}}\left(g_{2}\right)\right)=\rho\left(g_{1}, g_{2}\right) \quad \forall g_{0}, g_{1}, g_{2} \in \mathbb{G}
$$

and

$$
\rho\left(\delta_{\lambda}\left(g_{1}\right), \delta_{\lambda}\left(g_{2}\right)\right)=\lambda \rho\left(g_{1}, g_{2}\right) \quad \forall g_{1}, g_{2} \in \mathbb{G}, \forall \lambda>0 .
$$

It can be shown that $\rho$ induces the manifold topology on $\mathbb{G}$ and that $(\mathbb{G}, \rho)$ is a boundedly compact metric space. Moreover, it is easy to prove that left-invariant, homogeneous metrics $\rho_{1}$ and $\rho_{2}$ on $\mathbb{G}$ which both induce the manifold topology are equivalent in the sense that

$$
\frac{1}{c} \rho_{2}\left(g_{1}, g_{2}\right) \leq \rho_{1}\left(g_{1}, g_{2}\right) \leq c \rho_{2}\left(g_{1}, g_{2}\right) \quad \forall g_{1}, g_{2} \in \mathbb{G}
$$

for some constant $1 \leq c<+\infty$. We call a left-invariant, homogeneous metric on $\mathbb{G}$ which induces the manifold topology an intrinsic metric. Throughout the article, we fix a left-invariant inner product $\langle\cdot, \cdot\rangle$ on the horizontal bundle $\mathrm{H} \mathbb{G}$ and we denote by $\rho$ the induced Carnot-Carathéodory metric. The open metric ball of radius $r$ centered at $g \in \mathbb{G}$ is denoted $B(g, r)$.

Standard considerations show that $Q$-dimensional Hausdorff measure $\mathcal{H}^{Q}$ built with respect to $\rho$ is a bi-invariant Haar measure on $\mathbb{G}$ which is $Q$-homogeneous with respect to the dilations, that is

$$
\mathcal{H}^{Q}\left(l_{g_{0}}(A)\right)=\mathcal{H}^{Q}\left(r_{g_{0}}(A)\right)=\mathcal{H}^{Q}(A) \quad \forall A \subseteq \mathbb{G}, \forall g_{0} \in \mathbb{G}
$$

and

$$
\mathcal{H}^{Q}\left(\delta_{\lambda}(A)\right)=\lambda^{Q_{\mathcal{H}}^{Q}}(A) \quad \forall A \subseteq \mathbb{G}, \forall \lambda>0
$$

Let $\mathbb{G}$ be a Carnot group and let $\oplus_{i=1}^{s} V_{i}$ be a stratification of its Lie algebra $\mathfrak{g}$ of leftinvariant vector fields. A basis $\left(X_{1}, \ldots, X_{d}\right)$ of $\mathfrak{g}$ is said to be adapted to the stratification if $\left(X_{n_{j-1}+1}, \ldots, X_{n_{j}}\right)$ is a basis of $V_{j}$ for all $1 \leq j \leq s$, where $n_{j}=\sum_{i=1}^{j} d_{i}$ for $j=0,1, \ldots, s$. It will be convenient to work with a more explicit representation of a given Carnot group $\mathbb{G}$. Such representation can be obtained as follows: By (2.1), $*: \mathfrak{g} \times \mathfrak{g} \rightarrow \mathfrak{g}$ defines a group structure on $\mathfrak{g}$, and $\exp :(\mathfrak{g}, *) \rightarrow(\mathbb{G}, \cdot)$ is a Lie group isomorphism. Given an adapted basis $\left(X_{1}, \ldots, X_{d}\right)$ of $\mathfrak{g}$, we use the induced $\mathbb{R}$-linear mapping

$$
f: \mathfrak{g} \rightarrow \mathbb{R}^{d}, f\left(\sum_{i=1}^{d} x_{i} X_{i}\right)=\left(x_{1}, \ldots, x_{d}\right)
$$


to transport the group operation $*$ to $\mathbb{R}^{d}$. Then $\left(\mathbb{R}^{d}, *\right)$ is a Lie group isomorphic with $(\mathbb{G}, \cdot)$. Given $1 \leq i \leq d$, we let $\operatorname{deg}(i)=\min \left\{1 \leq k \leq s \mid i \leq \sum_{j=1}^{k} d_{j}\right\}$. Then

$$
x * x^{\prime}=x+x^{\prime}+P\left(x, x^{\prime}\right) \quad \forall x, x^{\prime} \in \mathbb{R}^{d},
$$

where $P_{i}\left(x, x^{\prime}\right)$ is a polynomial in the variables $x_{k}, x_{l}^{\prime}$ with $\operatorname{deg}(k), \operatorname{deg}(l)<\operatorname{deg}(i)$ if $d_{1}<i \leq d$, and $P_{i}\left(x, x^{\prime}\right)=0$ if $1 \leq i \leq d_{1}$. The unit element in $\left(\mathbb{R}^{d}, *\right)$ is 0 , and the inverse of $x \in \mathbb{R}^{d}$ with respect to $*$ is $-x$. Dilation with $\lambda>0$ is given by

$$
\delta_{\lambda}\left(x_{1}, \ldots, x_{d}\right)=\left(\lambda^{\operatorname{deg}(1)} x_{1}, \lambda^{\operatorname{deg}(2)} x_{2}, \ldots, \lambda^{\operatorname{deg}(d)} x_{d}\right) .
$$

The induced horizontal subbundle $H \mathbb{R}^{d}$ is spanned by the left-invariant vector fields $X$ on $\left(\mathbb{R}^{d}, *\right)$ uniquely determined by the condition

$$
X(0)=\sum_{i=1}^{d_{1}} x_{i} \partial_{i}(0)
$$

where $x_{1}, \ldots, x_{d_{1}} \in \mathbb{R}$ are arbitrary. The integral curve $\gamma: \mathbb{R} \rightarrow \mathbb{R}^{d}$ of the left-invariant vector field $X$ which satisfies the initial condition $\gamma(0)=x \in \mathbb{R}^{d}$ is given by

$$
\gamma(t)=x * \delta_{t}\left(x_{1}, \ldots, x_{d_{1}}, 0, \ldots, 0\right) \quad \forall t \in \mathbb{R} .
$$

It can be shown that the restriction of $\gamma$ to $\left[t_{1}, t_{2}\right]$ minimizes the sub-Riemannian length of admissible curves connecting $\gamma\left(t_{1}\right)$ with $\gamma\left(t_{2}\right)$ for all $t_{1}, t_{2} \in \mathbb{R}$ with $t_{1} \leq t_{2} . \gamma$ is a parametrization proportional to intrinsic arc length of $\gamma(\mathbb{R})$.

Finally, it follows from (2.3) and (2.4) that $d$-dimensional Hausdorff measure $\mathcal{H}_{E}^{d}$ built with respect to the Euclidean distance on $\mathbb{R}^{d}$ is a bi-invariant, $Q$-homogeneous [with respect to the dilations $(2.4)]$ Haar measure on $\left(\mathbb{R}^{d}, *\right)$. In particular, there exists a unique constant $0<\alpha<+\infty$ such that $\mathcal{H}^{Q}=\alpha \mathcal{H}_{E}^{d}$.

In the following, the notation $\mathbb{G} \equiv\left(\mathbb{R}^{d}, *\right)$ indicates that an adapted basis of the Lie algebra of $\mathbb{G}$ has been chosen and that the group operation of $\mathbb{G}$ has been transported to $\mathbb{R}^{d}$ by means of the procedure described above. If $\mathbb{G} \equiv\left(\mathbb{R}^{d}, *\right)$, then $(\cdot, \cdot)$ denotes the standard inner product on $\mathbb{R}^{d},\|\cdot\|$ the induced Euclidean norm and $B_{E}(x, r)$ the open ball of radius $r$ centered at $x \in \mathbb{R}^{d}$ with respect to the Euclidean metric $\rho_{E}$.

\subsection{Horizontal convexity}

The following definition of convexity in Carnot groups corresponds to the notion of "weak H-convexity" introduced by Danielli, Garofalo, and Nhieu in [5].

Definition 2.2. Let $\mathbb{G}$ be a Carnot group. A subset $C \subseteq \mathbb{G}$ is said to be h-convex if $\gamma([a, b]) \subseteq$ $C$ whenever $\gamma:[a, b] \rightarrow \mathbb{G}$ is an integral curve of some left-invariant, horizontal vector field and $\gamma(a), \gamma(b) \in C$. If $A \subseteq \mathbb{G}$ is any subset, the h-convex closure $\mathcal{C}(A)$ of $A$ is the smallest h-convex set which contains $A$.

A function $u: C \rightarrow \mathbb{R}$ defined on some h-convex subset $C \subseteq \mathbb{G}$ is said to be h-convex if $u \circ \gamma:[a, b] \rightarrow \mathbb{R}$ is convex (in the Euclidean sense) whenever $\gamma:[a, b] \rightarrow C$ is a parametrization proportional to intrinsic arc length of a segment of an integral curve of some left-invariant, horizontal vector field. 
The proof of the following proposition is a verification, and we can leave it to the reader to supply the details.

Proposition 2.3. Let $\mathbb{G}$ be a Carnot group, $C \subseteq \mathbb{G}$ an h-convex subset, $u, v: C \rightarrow \mathbb{R}$ $h$-convex functions, $g \in \mathbb{G}, \lambda>0$ and $c \geq 0$. Then

(i) $l_{g^{-1}}(C)$ is h-convex and $u \circ l_{g}: l_{g^{-1}}(C) \rightarrow \mathbb{R}$ is $h$-convex,

(ii) $\delta_{1 / \lambda}(C)$ is $h$-convex and $u \circ \delta_{\lambda}: \delta_{1 / \lambda}(C) \rightarrow \mathbb{R}$ is h-convex,

(iii) $c \cdot u: C \rightarrow \mathbb{R}$ is h-convex and

(iv) $u+v: C \rightarrow \mathbb{R}$ is h-convex.

Moreover,

(v) the intersection of a collection of $h$-convex subsets of $\mathbb{G}$ is $h$-convex,

(vi) $l_{g}(\mathcal{C}(A))=\mathcal{C}\left(l_{g}(A)\right)$ for all $A \subseteq \mathbb{G}$ and $g \in \mathbb{G}$, and

(vii) $\delta_{\lambda}(\mathcal{C}(A))=\mathcal{C}\left(\delta_{\lambda}(A)\right)$ for all $A \subseteq \mathbb{G}$ and $\lambda>0$.

Given a Carnot group $\mathbb{G} \equiv\left(\mathbb{R}^{d}, *\right)$, we say that a subset $C \subseteq \mathbb{R}^{d}$ is E-convex if it is convex in the usual Euclidean sense. Similarly, a function $f: C \rightarrow \mathbb{R}$ defined on an E-convex subset $C \subseteq \mathbb{R}^{d}$ is E-convex if it is convex in the Euclidean sense.

Proposition 2.4. In a Carnot group $\mathbb{G} \equiv\left(\mathbb{R}^{d}, *\right)$ of step at most two, any $E$-convex set $C \subseteq \mathbb{R}^{d}$ is $h$-convex and any E-convex function $u: C \rightarrow \mathbb{R}$ is $h$-convex.

Proof. The claim is an immediate consequence of the following well-known fact: If $\gamma:[a, b] \rightarrow\left(\mathbb{R}^{d}, *\right)$ is a parametrization proportional to intrinsic arc length of a segment of an integral curve of some left-invariant, horizontal vector field, then $\gamma([a, b])$ is a line segment and $\gamma$ is a parametrization proportional to Euclidean arc length of this line segment.

The converse of Proposition 2.4 is of course wrong. We refer the reader to [5] or [2]. The condition on the step of the group in Proposition 2.4 cannot be relaxed. Indeed, in the Engel group $\mathbb{E} \equiv\left(\mathbb{R}^{4}, *\right)$ (compare Section 5), the function

$$
u: \mathbb{E} \rightarrow \mathbb{R}, f\left(x_{1}, x_{2}, y, z\right)=z
$$

is not $\mathrm{h}$-convex.

Proposition 2.5. Let $\mathbb{G} \equiv\left(\mathbb{R}^{d}, *\right)$ be a Carnot group. There exists a constant $r_{0}>0$ such that, whenever $0<r<r_{0}$ and $\gamma: \mathbb{R} \rightarrow \mathbb{R}^{d}$ is an integral curve of some left-invariant, horizontal vector field with $\gamma(0)=x \in B_{E}(0, r)$, then there exist exactly one positive time $t_{+}>0$ and one negative time $t_{-}<0$ such that $\gamma\left(t_{+}\right), \gamma\left(t_{-}\right) \in \partial B_{E}(0, r)$.

Proof. Let $b \geq 1$ such that $\left\|\gamma^{\prime \prime}(t)\right\| \leq b$ on $[-1,1]$ whenever $\gamma$ is a parametrization by intrinsic arc length of an integral curve of some left-invariant, horizontal vector field, and $\gamma$ satisfies the initial condition $\gamma(0) \in B_{E}(0,1)$. Define $r_{0}=(\sqrt{5}-2) /(8 b)$. Fix $0<r<r_{0}$ and $x \in B_{E}(0, r)$. If $v \in \mathbb{R}^{d_{1}} \times\{0\} \subseteq \mathbb{R}^{d},\|v\|=1$, then $\gamma: \mathbb{R} \rightarrow \mathbb{R}^{d}, \gamma(t)=x * \delta_{t}(v)$ is a parametrization by intrinsic arc length of an integral curve of the left-invariant, horizontal vector field $X$ uniquely determined by the condition $X(0)=\sum_{i=1}^{d_{1}} v_{i} \partial_{i}(0)$, and $\gamma$ satisfies the initial condition $\gamma(0)=x$. If $\pi: \mathbb{R}^{d_{1}} \times \mathbb{R}^{d^{-d_{1}}} \rightarrow \mathbb{R}^{d_{1}}$ is orthogonal projection, then

$$
\|\gamma(t)\| \geq\|\pi(\gamma(t))\|=\left\|\left(x_{1}, \ldots, x_{d_{1}}\right)+t\left(v_{1}, \ldots, v_{d_{1}}\right)\right\| \geq|t|-\left\|\left(x_{1}, \ldots, x_{d_{1}}\right)\right\| .
$$


Hence, $|t| \geq 2 r$ implies $\gamma(t) \notin \bar{B}_{E}(0, r)$. Now let

$$
t_{+}=\inf \left\{t>0 \mid \gamma(t) \notin B_{E}(0, r)\right\} .
$$

Then $0<t_{+}<2 r, \gamma\left(t_{+}\right) \in \partial B_{E}(0, r), \gamma(t) \in B_{E}(0, r)$ for $0 \leq t<t_{+}$, and if $n$ denotes the unit outer normal to $\partial B_{E}(0, r)$ at $\gamma\left(t_{+}\right)$, then $\left(\gamma^{\prime}\left(t_{+}\right), n\right) \geq 0$. We have to show that $\gamma(t) \notin \bar{B}_{E}(0, r)$ when $t_{+}<t<2 r$. We compute

$$
\begin{aligned}
\|\gamma(t)\| & =\left\|\gamma\left(t_{+}\right)+\left(t-t_{+}\right) \gamma^{\prime}\left(t_{+}\right)+\left(t-t_{+}\right)^{2} \int_{0}^{1}(1-s) \gamma^{\prime \prime}\left(t_{+}+s\left(t-t_{+}\right)\right) d s\right\| \\
& \geq\left\|\gamma\left(t_{+}\right)+\left(t-t_{+}\right) \gamma^{\prime}\left(t_{+}\right)\right\|-\left(t-t_{+}\right)^{2} \int_{0}^{1}\left\|(1-s) \gamma^{\prime \prime}\left(t_{+}+s\left(t-t_{+}\right)\right)\right\| d s \\
& \geq\left(r^{2}+\left(t-t_{+}\right)^{2}\left\|\gamma^{\prime}\left(t_{+}\right)\right\|^{2}\right)^{\frac{1}{2}}-\left(t-t_{+}\right)^{2} b \\
& \geq\left(r^{2}+\left(t-t_{+}\right)^{2}\right)^{\frac{1}{2}}-\left(t-t_{+}\right)^{2} b \\
& =r+\frac{\left(t-t_{+}\right)^{2}}{2 r}+\left(t-t_{+}\right)^{4} \int_{0}^{1} \frac{(-1)(1-s)}{4\left(r^{2}+s\left(t-t_{+}\right)^{2}\right)^{\frac{3}{2}}} d s-\left(t-t_{+}\right)^{2} b .
\end{aligned}
$$

If $t-t_{+} \leq r / 2$, then

$$
\begin{aligned}
\|\gamma(t)\| & \geq r+\frac{\left(t-t_{+}\right)^{2}}{2 r}-\frac{\left(t-t_{+}\right)^{4}}{4 r^{3}}-\left(t-t_{+}\right)^{2} b \\
& \geq r+\frac{\left(t-t_{+}\right)^{2}}{2 r}-\frac{\left(t-t_{+}\right)^{2}}{16 r}-\frac{\left(t-t_{+}\right)^{2}}{8 r}>r .
\end{aligned}
$$

On the other hand, if $t-t_{+}>r / 2$, then

$$
\|\gamma(t)\| \geq\left(r^{2}+\left(t-t_{+}\right)^{2}\right)^{\frac{1}{2}}-\left(t-t_{+}\right)^{2} b>\frac{\sqrt{5} r}{2}-(2 r)^{2} b>\frac{\sqrt{5} r}{2}-\frac{(\sqrt{5}-2) r}{2}=r .
$$

The proof for the negative time $t_{-}<0$ is analogous.

Corollary 2.6. Let $\mathbb{G} \equiv\left(\mathbb{R}^{d}, *\right)$ be a Carnot group and $0<r<r_{0}$, where $r_{0}$ is given by Proposition 2.5. Then $B_{E}(0, r)$ is h-convex. Consequently, by Proposition 2.3, there exists a countable basis of the topology consisting of bounded, $h$-convex sets with smooth boundary.

The reader familiar with the first Heisenberg group $\mathbb{H}_{1}$ can convince himself that the open ball $B(0,1) \subseteq \mathbb{H}_{1}$ is not h-convex.

Proposition 2.7. Let $\mathbb{G} \equiv\left(\mathbb{R}^{d}, *\right)$ be a Carnot group, $b$ and $r_{0}=(\sqrt{5}-2) /(8 b)$ the constants appearing in Proposition 2.5 and its proof, and let $u \in C^{2}\left(B_{E}\left(0, r_{0}\right)\right)$ be a uniformly strictly $E$-convex function. Suppose that $\lambda>0$ is a lower bound for the eigenvalues of $D^{2} u$ in $B_{E}\left(0, r_{0}\right)$ and suppose that $0<M<+\infty$ is an upper bound for $\|\nabla u\|$ in $B_{E}\left(0, r_{0}\right)$. Then there exists $0<r_{1}=r_{1}(\lambda, M)<r_{0}$ such that

$$
v: B_{E}(0, r) \rightarrow \mathbb{R}, \quad v(x)=u\left(\frac{r_{0}}{r} x\right)
$$

is $h$-convex whenever $0<r<r_{1}$.

Proof. By Corollary 2.6, $B_{E}(0, r)$ is h-convex for $0<r<r_{0}$. Let $\gamma:\left[t_{-}, t_{+}\right] \rightarrow B_{E}(0, r)$ be a parametrization by intrinsic arc length of a segment of an integral curve of some left-invariant, 
horizontal vector field. We can assume $-1 \leq t_{-}<t_{+} \leq 1$ and $\left\|\gamma^{\prime \prime}(t)\right\| \leq b$ for all $t \in\left[t_{-}, t_{+}\right]$ (cf. the proof of Proposition 2.5). Then, for each $t \in\left[t_{-}, t_{+}\right]$, we get

$$
\begin{aligned}
(v \circ \gamma)^{\prime \prime}(t) & =\left(D^{2} v(\gamma(t)) \gamma^{\prime}(t), \gamma^{\prime}(t)\right)+\left(\nabla v(\gamma(t)), \gamma^{\prime \prime}(t)\right) \\
& =\left(\frac{r_{0}}{r}\right)^{2}\left(D^{2} u\left(\frac{r_{0}}{r} \gamma(t)\right) \gamma^{\prime}(t), \gamma^{\prime}(t)\right)+\frac{r_{0}}{r}\left(\nabla u\left(\frac{r_{0}}{r} \gamma(t)\right), \gamma^{\prime \prime}(t)\right) \\
& \geq\left(\frac{r_{0}}{r}\right)^{2} \lambda\left\|\gamma^{\prime}(t)\right\|^{2}-\frac{r_{0}}{r}\left\|\nabla u\left(\frac{r_{0}}{r} \gamma(t)\right)\right\|\left\|\gamma^{\prime \prime}(t)\right\| \\
& \geq\left(\frac{r_{0}}{r}\right)^{2} \lambda-\frac{r_{0}}{r} M b .
\end{aligned}
$$

\section{Lipschitz continuity of bounded above h-convex functions}

In this section, we show than an h-convex function which is locally bounded above is locally Lipschitz continuous with respect to any intrinsic metric. This result can be found in [15], for instance. Nevertheless, we give the proof below.

Lemma 3.1. Let $\mathbb{G}$ be a Carnot group, $\Omega \subseteq \mathbb{G}$ an $h$-convex, open subset and $u: \Omega \rightarrow \mathbb{R}$ an $h$-convex function. Suppose that $u$ is locally bounded above. Then $u$ is locally bounded below.

Proof. Let $g_{0} \in \Omega$ and $r>0$ such that $B\left(g_{0}, 4 r\right) \Subset \Omega$ and $u$ is bounded above in $\bar{B}\left(g_{0}, 4 r\right)$, say $u \leq M$ in $\bar{B}\left(g_{0}, 4 r\right)$ for some $M \geq 0$. We claim that there exist $l=l(\mathbb{G}) \in \mathbb{N}$ and $n=n(\mathbb{G}) \in \mathbb{N}$ such that $g_{1} \in B\left(g_{0}, r\right)$ and $u\left(g_{1}\right) \leq-4^{l} m$ implies $u \leq-m$ in $B\left(g_{1}, r / l n\right)$ for all $m \geq 2 M$. Notice that this gives the lemma since then, on the one hand,

$$
\mathcal{H}^{Q}\left(\left\{g \in B\left(g_{0}, 4 r\right) \mid u(g)>-m\right\}\right) \leq \mathcal{H}^{Q}\left(B\left(g_{0}, 4 r\right)\right)-\mathcal{H}^{Q}\left(B\left(g_{1}, r /(l n)\right)\right),
$$

while, on the other hand,

$$
\mathcal{H}^{Q}\left(\left\{g \in B\left(g_{0}, 4 r\right) \mid u(g) \geq-m\right\}\right)>\mathcal{H}^{Q}\left(B\left(g_{0}, 4 r\right)\right)-\mathcal{H}^{Q}\left(B\left(g_{1}, r /(l n)\right)\right)
$$

when $m$ is sufficiently large. This contradiction forces $u \geq-4^{l} m$ in $B\left(g_{0}, r\right)$ for sufficiently large $m$.

Let $\gamma: \mathbb{R} \rightarrow \mathbb{G}$ be a parametrization by intrinsic arc length of an integral curve of some left-invariant, horizontal vector field, and suppose that $\gamma$ satisfies the initial condition $\gamma(0) \in$ $B\left(g_{0}, 2 r\right)$. Define

$$
t_{-}=\max \left\{t<0 \mid \gamma(t) \in \partial B\left(g_{0}, 4 r\right)\right\}
$$

and

$$
t_{+}=\min \left\{t>0 \mid \gamma(t) \in \partial B\left(g_{0}, 4 r\right)\right\} .
$$

We have $t_{-} \geq-6 r$ and $t_{+} \leq 6 r$. Let $t \in\left[t_{-}, t_{+}\right]$such that $\gamma(t) \in B\left(g_{0}, 2 r\right)$. If $t \geq 0$, then $t=(1-\lambda) \overline{0}+\lambda t_{+}$with $\lambda \leq \frac{2}{3}$. Suppose that $u(\gamma(0)) \leq-4^{j+1} m$. Then the convexity of $u \circ \gamma:\left[t_{-}, t_{+}\right] \rightarrow \mathbb{G}$ implies

$$
\begin{aligned}
u(\gamma(t)) & \leq(1-\lambda) u(\gamma(0))+\lambda u\left(\gamma\left(t_{+}\right)\right) \leq-\frac{1}{3} 4^{j+1} m+\frac{2}{3} M \\
& \leq\left(-\frac{4}{3}+\frac{4^{-j}}{3}\right) 4^{j} m \leq-4^{j} m .
\end{aligned}
$$


Similarly, $u(\gamma(t)) \leq-4^{j} m$ if $t \leq 0$ and $u(\gamma(0)) \leq-4^{j+1} m$. This shows that if $S \subseteq B\left(g_{0}, 2 r\right)$ is a segment of an integral curve of some left-invariant, horizontal vector field and if $u\left(g_{1}\right) \leq$ $-4^{j+1} m$ for some $g_{1} \in S$, then $u \leq-4^{j} m$ on the whole segment $(j \in \mathbb{N} \cup\{0\}, m \geq 2 M)$. By [10, Lemma 1.40], there exist constants $l=l(\mathbb{G}) \in \mathbb{N}$ and $n=n(\mathbb{G}) \in \mathbb{N}$ with the following property: Any pair of points $g_{1}, g_{2} \in \mathbb{G}$ can be connected by a path consisting of at most $l$ segments of integral curves of left-invariant, horizontal vector fields, such that each segment has length at most $n \rho\left(g_{1}, g_{2}\right)$. Thus, if $g_{1} \in B\left(g_{0}, r\right)$ and $u\left(g_{1}\right) \leq-4^{l} m$, then $u(g) \leq-m$ for each $g \in B\left(g_{1}, r /(\ln )\right)$.

Proposition 3.2. Let $\mathbb{G}$ be a Carnot group, $\Omega \subseteq \mathbb{G}$ an h-convex, open set and $u: \Omega \rightarrow \mathbb{R}$ an $h$-convex function. Suppose that $u$ is locally bounded. Then $u$ is locally Lipschitz continuous with respect to any intrinsic metric on $\mathbb{G}$.

Proof. Let $g_{0} \in \Omega$ and $r>0$ such that $B\left(g_{0}, 2 r\right) \Subset \Omega$ and $u$ is bounded in $\bar{B}\left(g_{0}, 2 r\right)$, say $|u| \leq M$ in $\bar{B}\left(g_{0}, 2 r\right)$ for some $M \geq 0$. Let $\gamma: \mathbb{R} \rightarrow \mathbb{G}$ be a parametrization by intrinsic arc length of an integral curve of some left-invariant, horizontal vector field and suppose that $\gamma$ satisfies the initial condition $\gamma(0) \in B\left(g_{0}, r\right)$. Define

$$
t_{-}=\max \left\{t<0 \mid \gamma(t) \in \partial B\left(g_{0}, 2 r\right)\right\}
$$

and

$$
t_{+}=\min \left\{t>0 \mid \gamma(t) \in \partial B\left(g_{0}, 2 r\right)\right\} .
$$

We have $2 r \leq t_{+}-t_{-} \leq 4 r$, and if $t \in\left[t_{-}, t_{+}\right]$and $\gamma(t) \in B\left(g_{0}, r\right)$, then $t-t_{-} \geq r$ and $t_{+}-t \geq r$. Hence, $t=(1-\lambda) t_{-}+\lambda t_{+}$, where $\lambda \in[1 / 4,3 / 4]$. Now let $t_{1}, t_{2} \in\left[t_{-}, t_{+}\right]$such that $t_{1}<t_{2}$ and $\gamma\left(t_{1}\right), \gamma\left(t_{2}\right) \in B\left(g_{0}, r\right)$. Then

$$
t_{1}=\left(1-\lambda_{1}\right) t_{-}+\lambda_{1} t_{+} \quad \text { and } t_{2}=\left(1-\lambda_{2}\right) t_{-}+\lambda_{2} t_{+},
$$

where $\lambda_{1}, \lambda_{2} \in[1 / 4,3 / 4]$, and $\lambda_{1}<\lambda_{2}$. Thus,

$$
t_{1}=\frac{\lambda_{2}-\lambda_{1}}{\lambda_{2}} t_{-}+\frac{\lambda_{1}}{\lambda_{2}} t_{2} \quad \text { and } \quad t_{2}=\frac{1-\lambda_{2}}{1-\lambda_{1}} t_{1}+\frac{\lambda_{2}-\lambda_{1}}{1-\lambda_{1}} t_{+} .
$$

The convexity of $u \circ \gamma:\left[t_{-}, t_{+}\right] \rightarrow \mathbb{R}$ implies

$$
\begin{aligned}
u\left(t_{1}\right)-u\left(t_{2}\right) & \leq \frac{\lambda_{2}-\lambda_{1}}{\lambda_{2}} u\left(t_{-}\right)+\frac{\lambda_{1}-\lambda_{2}}{\lambda_{2}} u\left(t_{2}\right) \\
& =\frac{\rho\left(\gamma\left(t_{1}\right), \gamma\left(t_{2}\right)\right)}{\lambda_{2}\left(t_{+}-t_{-}\right)} u\left(t_{-}\right)-\frac{\rho\left(\gamma\left(t_{1}\right), \gamma\left(t_{2}\right)\right)}{\lambda_{2}\left(t_{+}-t_{-}\right)} u\left(t_{2}\right) \\
& \leq \frac{8 M}{r} \rho\left(\gamma\left(t_{1}\right), \gamma\left(t_{2}\right)\right)
\end{aligned}
$$

and

$$
\begin{aligned}
u\left(t_{2}\right)-u\left(t_{1}\right) & \leq \frac{\lambda_{1}-\lambda_{2}}{1-\lambda_{1}} u\left(t_{1}\right)+\frac{\lambda_{2}-\lambda_{1}}{1-\lambda_{1}} u\left(t_{+}\right) \\
& =-\frac{\rho\left(\gamma\left(t_{1}\right), \gamma\left(t_{2}\right)\right)}{\left(1-\lambda_{1}\right)\left(t_{+}-t_{-}\right)} u\left(t_{1}\right)+\frac{\rho\left(\gamma\left(t_{1}\right), \gamma\left(t_{2}\right)\right)}{\left(1-\lambda_{1}\right)\left(t_{+}-t_{-}\right)} u\left(t_{+}\right) \\
& \leq \frac{8 M}{r} \rho\left(\gamma\left(t_{1}\right), \gamma\left(t_{2}\right)\right) .
\end{aligned}
$$

We have shown that

$$
\left|u\left(g_{1}\right)-u\left(g_{2}\right)\right| \leq \frac{8 M}{r} \rho\left(g_{1}, g_{2}\right) \quad \forall g_{1}, g_{2} \in S
$$


whenever $S \subseteq B\left(g_{0}, r\right)$ is a segment of an integral curve of some left-invariant vector field. By [10, Lemma 1.40], there exist constants $l=l(\mathbb{G}) \in \mathbb{N}$ and $n=n(\mathbb{G}) \in \mathbb{N}$ with the following property: Any pair of points $g_{1}, g_{2} \in \mathbb{G}$ can be connected by a path consisting of at most $l$ segments of integral curves of left-invariant, horizontal vector fields, such that each segment has length at most $n \rho\left(g_{1}, g_{2}\right)$. In particular, if we let $\sigma=r /(2 l n+1)$ and $g_{1}, g_{2} \in B\left(g_{0}, \sigma\right)$, then (3.1) implies $\left|u\left(g_{1}\right)-u\left(g_{2}\right)\right| \leq L \rho\left(g_{1}, g_{2}\right)$ with $L=8 M \ln / r$.

\section{Local upper bounds for h-convex functions in step two}

This section is devoted to the proof of Theorem 1.2. Notice that in view of the results of the previous section, Theorem 1.1 is an immediate consequence of Theorem 1.2 and of the following lemma.

Lemma 4.1. Let $\mathbb{G}$ be a Carnot group. Suppose there exists a finite subset $F \subseteq \mathbb{G}$ whose $h$-convex closure $\mathcal{C}(F)$ has nonempty interior. Then any h-convex function $u: \Omega \rightarrow \mathbb{R}$ defined on an $h$-convex, open subset $\Omega \subseteq \mathbb{G}$ is locally bounded above.

Proof. Let $\mathcal{I}$ denote the set of parameterizations $\gamma:[0,1] \rightarrow \mathbb{G}$ proportional to intrinsic arc length of bounded, closed segments of integral curves of left-invariant, horizontal vector fields. Given $A \subseteq \mathbb{G}$ we let

$$
\begin{aligned}
\mathrm{H}(A) & =\{\gamma(t) \mid \gamma \in \mathcal{I}, t \in[0,1], \gamma(0), \gamma(1) \in A\}, \\
\mathrm{H}^{0}(A) & =A, \\
\mathrm{H}^{k+1}(A) & =\mathrm{H}\left(\mathrm{H}^{k}(A)\right) \quad \text { for all } k \in \mathbb{N}_{0} \quad \text { and } \\
\mathrm{H}^{\infty}(A) & =\bigcup_{k \in \mathbb{N}_{0}} \mathrm{H}^{k}(A) .
\end{aligned}
$$

We have $\mathcal{C}(A)=\mathrm{H}^{\infty}(A), l_{g}(\mathrm{H}(A))=\mathrm{H}\left(l_{g}(A)\right)$ for all $g \in \mathbb{G}, \delta_{\lambda}(\mathrm{H}(A))=\mathrm{H}\left(\delta_{\lambda}(A)\right)$ for all $\lambda>0$ and $\mathrm{H}(A)$ is compact if $A$ is.

The compactness property of the operator $\mathrm{H}$ and the Theorem of Baire imply that $\mathrm{H}^{k}(F)$ has nonempty interior for some $k=k(\mathbb{G}) \in \mathbb{N}$. Since $\mathrm{H}$-and thus $\mathrm{H}^{k}$-commutes with left translations and dilations, it follows that if $\Omega \subseteq \mathbb{G}$ is any h-convex, open subset, then for each $g_{0} \in \Omega$ there exists a finite subset $F\left(g_{0}\right) \subseteq \Omega$ such that $g_{0}$ is contained in the interior of $\mathrm{H}^{k}\left(F\left(g_{0}\right)\right)$. Finally, if $u: \Omega \rightarrow \mathbb{R}$ is h-convex, then $u \leq \max \left\{u(g) \mid g \in F\left(g_{0}\right)\right\}$ in $\mathrm{H}^{j}\left(F\left(g_{0}\right)\right)$ for all $1 \leq j \leq k$ by induction, using the convexity of $u \circ \gamma$ for $\gamma \in \mathcal{I}$. In particular, $u \leq$ $\max \left\{u(g) \mid g \in F\left(g_{0}\right)\right\}$ in the interior of $\mathrm{H}^{k}\left(F\left(g_{0}\right)\right)$.

Lemma 4.1 motivates the following definition.

Definition 4.2. We say that a Carnot group $\mathbb{G}$ is finitely h-convex if it contains a finite subset $F \subseteq \mathbb{G}$ whose h-convex closure $\mathcal{C}(F)$ has nonempty interior.

The remainder of this section is devoted to the proof of Theorem 1.2. The proof is by induction on the dimension $d_{1}$ of the generating layer in the Lie algebra $\mathfrak{g}$ of $\mathbb{G}$. We will need the following lemma in order to perform the induction step.

Lemma 4.3. Let $\mathbb{G} \equiv\left(\mathbb{R}^{d}, *\right) \equiv\left(\mathbb{R}^{d_{1}} \times \mathbb{R}^{d_{2}}, *\right)$ be a Carnot group of step two and let $\left(X_{1}, \ldots, X_{d_{1}}, Y_{1}, \ldots, Y_{d_{2}}\right)$ be a basis of the Lie algebra $\mathfrak{g}$ of left-invariant vector fields on $\mathbb{G}$, adapted to the given stratification $\mathfrak{g}=V_{1} \oplus V_{2}$. Suppose that for some $2 \leq k \leq d_{1}$, the following hypotheses are verified: 
(i) The Lie subalgebra generated by $X_{1}, \ldots, X_{k}$ is contained in

$$
\operatorname{span}_{\mathbb{R}}\left\{X_{1}, \ldots, X_{k}\right\} \oplus \operatorname{span}_{\mathbb{R}}\left\{Y_{1}, \ldots, Y_{l}\right\} .
$$

(ii) There exists a finite set $A_{0} \subseteq \mathbb{R}^{d_{1}} \times \mathbb{R}^{d_{2}}$ and a constant $K_{0}>0$ such that the set $B_{0}$ consisting of pairs $(0, y) \in \mathbb{R}^{d_{1}} \times \mathbb{R}^{d_{2}}$ with $\left|y_{j}\right| \leq K_{0}$ for $1 \leq j \leq l$ and $y_{j}=0$ for $l+1 \leq j \leq d_{2}$ is contained in $\mathcal{C}\left(A_{0}\right)$.

Then there exists a finite set $A_{k} \subseteq \mathbb{R}^{d_{1}} \times \mathbb{R}^{d_{2}}$ and a constant $K_{k}>0$ such that the set $B_{k}$ consisting of pairs $(x, y) \in \mathbb{R}^{d_{1}} \times \mathbb{R}^{d_{2}}$ with $\left|x_{i}\right| \leq K_{k}$ for $1 \leq i \leq k, x_{i}=0$ for $k+1 \leq i \leq d_{1},\left|y_{j}\right| \leq K_{k}$ for $1 \leq j \leq l$ and $y_{j}=0$ for $l+1 \leq j \leq d_{2}$ is contained in $\mathcal{C}\left(A_{k}\right)$.

Proof. Let $K_{1}=K_{0}$ and $g=(x, 0)$ with $x_{1}=K_{1}$ and $x_{i}=0$ for $2 \leq i \leq d_{1}$. Define $A_{1}=l_{-g}\left(A_{0}\right) \cup l_{g}\left(A_{0}\right)$. By assumption,

$$
l_{-g}\left(B_{0}\right) \subseteq l_{-g}\left(\mathcal{C}\left(A_{0}\right)\right)=\mathcal{C}\left(l_{-g}\left(A_{0}\right)\right), \quad l_{g}\left(B_{0}\right) \subseteq l_{g}\left(\mathcal{C}\left(A_{0}\right)\right)=\mathcal{C}\left(l_{g}\left(A_{0}\right)\right)
$$

whence

$$
\begin{aligned}
l_{-g}\left(B_{0}\right) \cup l_{g}\left(B_{0}\right) & \subseteq \mathcal{C}\left(l_{-g}\left(A_{0}\right)\right) \cup \mathcal{C}\left(l_{g}\left(A_{0}\right)\right) \\
& \subseteq \mathcal{C}\left(l_{-g}\left(A_{0}\right) \cup l_{g}\left(A_{0}\right)\right)=\mathcal{C}\left(A_{1}\right) .
\end{aligned}
$$

For fixed $y_{1}, \ldots, y_{l} \in \mathbb{R}$ with $\left|y_{j}\right| \leq K_{1}$,

$$
\begin{aligned}
S & =\left\{\left(0, \ldots, 0, y_{1}, \ldots, y_{l}, 0, \ldots, 0\right) * \delta_{\lambda}(g) \mid \lambda \in[-1,1]\right\} \\
& =\left\{\left(\lambda, 0, \ldots, 0, y_{1}, \ldots, y_{l}, 0, \ldots, 0\right) \mid \lambda \in\left[-K_{1}, K_{1}\right]\right\}
\end{aligned}
$$

is a segment of an integral curve of a left-invariant, horizontal vector field, and the endpoints of $S$ are contained in $l_{-g}\left(B_{0}\right) \cup l_{g}\left(B_{0}\right)$. Thus, the set $B_{1}$ consisting of pairs $(x, y) \in \mathbb{R}^{d_{1}} \times \mathbb{R}^{d_{2}}$ with $\left|x_{1}\right| \leq K_{1}, x_{i}=0$ for $2 \leq i \leq d_{1},\left|y_{j}\right| \leq K_{1}$ for $1 \leq j \leq l$ and $y_{j}=0$ for $l+1 \leq j \leq d_{1}$ is contained in $\mathcal{C}\left(A_{1}\right)$.

Let $1 \leq \tilde{k}<k$ and suppose that there exist a finite set $A_{\tilde{k}} \subseteq \mathbb{G}$ and a constant $K_{\tilde{k}}>0$ such that the set $B_{\tilde{k}}$ consisting of pairs $(x, y) \in \mathbb{R}^{d_{1}} \times \mathbb{R}^{d_{2}}$ with $\left|x_{i}\right| \leq K_{\tilde{k}}$ for $1 \leq i \leq \tilde{k}, x_{i}=0$ for $\tilde{k}+1 \leq i \leq d_{1},\left|y_{j}\right| \leq K_{\tilde{k}}$ for $1 \leq j \leq l$ and $y_{j}=0$ for $l+1 \leq j \leq d_{2}$ is contained in $\mathcal{C}\left(A_{\tilde{k}}\right)$. Let $0<\epsilon \leq K_{\tilde{k}}$ and $g=(x, 0)$ with $x_{\tilde{k}+1}=\epsilon$ and $x_{i}=0$ for $1 \leq i \leq d_{1}$ and $i \neq \tilde{k}+1$. Define $A_{\tilde{k}+1}=l_{-g}\left(A_{\tilde{k}}\right) \cup l_{g}\left(A_{\tilde{k}}\right)$. By inductive hypothesis,

$$
l_{-g}\left(B_{\tilde{k}}\right) \subseteq l_{-g}\left(\mathcal{C}\left(A_{\tilde{k}}\right)\right)=\mathcal{C}\left(l_{-g}\left(A_{\tilde{k}}\right)\right)
$$

and

$$
l_{g}\left(B_{\tilde{k}}\right) \subseteq l_{g}\left(\mathcal{C}\left(A_{\tilde{k}}\right)\right)=\mathcal{C}\left(l_{g}\left(A_{\tilde{k}}\right)\right)
$$

whence

$$
\begin{aligned}
l_{-g}\left(B_{\tilde{k}}\right) \cup l_{g}\left(B_{\tilde{k}}\right) & \subseteq \mathcal{C}\left(l_{-g}\left(A_{\tilde{k}}\right)\right) \cup \mathcal{C}\left(l_{g}\left(A_{\tilde{k}}\right)\right) \\
& \subseteq \mathcal{C}\left(l_{-g}\left(A_{\tilde{k}}\right) \cup l_{g}\left(A_{\tilde{k}}\right)\right)=\mathcal{C}\left(A_{\tilde{k}+1}\right)
\end{aligned}
$$

Let

$$
h=\left(x_{1}, \ldots, x_{\tilde{k}}, 0, \ldots, 0, y_{1}, \ldots, y_{l}, 0, \ldots, 0\right) \in B_{\tilde{k}} .
$$

In view of hypothesis (i), we have

$$
(-g) * h=\left(x_{1}, \ldots, x_{\tilde{k}},-\epsilon, 0, \ldots, 0, y_{1}, \ldots, y_{l}, 0, \ldots, 0\right)-R_{\epsilon}\left(x_{1}, \ldots, x_{\tilde{k}}, \epsilon\right)
$$


and

$$
g * h=\left(x_{1}, \ldots, x_{\tilde{k}}, \epsilon, 0, \ldots, 0, y_{1}, \ldots, y_{l}, 0, \ldots, 0\right)+R_{\epsilon}\left(x_{1}, \ldots, x_{\tilde{k}}, \epsilon\right),
$$

where the first $d_{1}$ and last $d_{2}-l$ coordinates of $R_{\epsilon}\left(x_{1}, \ldots, x_{\tilde{k}}, \epsilon\right)$ vanish, and

$$
\left\|R_{\epsilon}\left(x_{1}, \ldots, x_{\tilde{k}}, \epsilon\right)\right\| \leq \beta K_{\tilde{k}} \epsilon
$$

for some constant $\beta=\beta(\mathbb{G})$. Hence, if we choose $\epsilon$ small enough, the pairs $(x, y) \in \mathbb{R}^{d_{1}} \times \mathbb{R}^{d_{2}}$ with $\left|x_{i}\right| \leq K_{\tilde{k}}$ for $1 \leq i \leq \tilde{k}, x_{\tilde{k}+1}=-\epsilon, x_{i}=0$ for $\tilde{k}+1 \leq i \leq d_{1},\left|y_{j}\right| \leq K_{\tilde{k}} / 2$ for $1 \leq j \leq l$ and $y_{j}=0$ for $l+1 \leq j \leq d_{2}$ are contained in $l_{-g}\left(B_{\tilde{k}}\right)$. Similarly, the pairs $(x, y) \in \mathbb{R}^{d_{1}} \times \mathbb{R}^{d_{2}}$ with $\left|x_{i}\right| \leq K_{\tilde{k}}$ for $1 \leq i \leq \tilde{k}, x_{\tilde{k}+1}=\epsilon, x_{i}=0$ for $\tilde{k}+1 \leq i \leq d_{1},\left|y_{j}\right| \leq K_{\tilde{k}} / 2$ for $1 \leq j \leq l$ and $y_{j}=0$ for $l+1 \leq j \leq d_{2}$ are contained in $l_{g}\left(B_{\tilde{k}}\right)$. For fixed

$$
h=\left(x_{1}, \ldots, x_{\tilde{k}}, 0, \ldots, 0, y_{1}, \ldots, y_{l}, 0, \ldots, 0\right)
$$

with $\left|x_{i}\right| \leq \sigma$ for $1 \leq i \leq \tilde{k}$ and $\left|y_{j}\right| \leq K_{\tilde{k}} / 4$ for $1 \leq j \leq l$,

$$
S=\left\{h * \delta_{\lambda}(g) \mid \lambda \in[-1,1]\right\}
$$

is a segment of an integral curve of a left-invariant, horizontal vector field. In view of hypothesis (i), we have

$$
h * \delta_{\lambda}(g)=\left(x_{1}, \ldots, x_{\tilde{k}}, \lambda \epsilon, 0, \ldots, 0, y_{1}, \ldots, y_{l}, 0, \ldots, 0\right)+R_{\sigma}\left(x_{1}, \ldots, x_{\tilde{k}}, \lambda\right),
$$

where the first $d_{1}$ and last $d_{2}-l$ coordinates of $R_{\sigma}\left(x_{1}, \ldots, x_{\tilde{k}}, \lambda\right)$ vanish, and

$$
\left\|R_{\sigma}\left(x_{1}, \ldots, x_{\tilde{k}}, \lambda\right)\right\| \leq \beta \sigma \epsilon .
$$

Hence, if $\sigma$ is sufficiently small, the endpoints of $S$ are contained in $l_{-g}\left(B_{\tilde{k}}\right) \cup l_{g}\left(B_{\tilde{k}}\right)$, whence $S \subseteq \mathcal{C}\left(A_{\tilde{k}+1}\right)$, and the union of such segments contains the set consisting of pairs $(x, y) \in$ $\mathbb{R}^{d_{1}} \times \mathbb{R}^{d_{2}}$ with

(i) $\left|x_{i}\right| \leq \sigma$ for $1 \leq i \leq \tilde{k}$,

(ii) $\left|x_{\tilde{k}+1}\right| \leq \epsilon$,

(iii) $x_{i}=0$ for $\tilde{k}+2 \leq i \leq d_{1}$

(iv) $\left|y_{j}\right| \leq \sigma$ for $1 \leq j \leq l$ and

(v) $y_{j}=0$ for $l+1 \leq j \leq d_{2}$.

Thus, if $K_{\tilde{k}+1}=\min \{\sigma, \epsilon\}$, then the set $B_{\tilde{k}+1}$ consisting of pairs $(x, y) \in \mathbb{R}^{d_{1}} \times \mathbb{R}^{d_{2}}$ with

(i) $\left|x_{i}\right| \leq K_{\tilde{k}+1}$ for $1 \leq i \leq \tilde{k}+1$,

(ii) $x_{i}=0$ for $\tilde{k}+2 \leq i \leq d_{1}$

(iii) $\left|y_{j}\right| \leq K_{\tilde{k}+1}$ for $1 \leq j \leq l$ and

(iv) $y_{j}=0$ for $l+1 \leq j \leq d_{2}$

is contained in $\mathcal{C}\left(A_{\tilde{k}+1}\right)$. This concludes the induction step and the proof.

Proof of Theorem 1.2. Let $\mathfrak{g}=V_{1} \oplus V_{2}$ be the given stratification of the Lie algebra of left-invariant vector fields on $\mathbb{G} \equiv\left(\mathbb{R}^{d}, *\right) \equiv\left(\mathbb{R}^{d_{1}} \times \mathbb{R}^{d_{2}}, *\right)$. Let $\left(X_{1}, \ldots, X_{d_{1}}\right)$ be a basis of $V_{1}$ such that $\left[X_{1}, X_{2}\right] \neq 0$. Set $l_{1}=0$. Clearly, we can find a basis $\left(Y_{1}, \ldots, Y_{d_{2}}\right)$ of $V_{2}$ with the following properties: 
(i) There exist integers $1=l_{2} \leq \ldots \leq l_{d_{1}}=d_{2}$ such that $\left(Y_{1}, \ldots, Y_{l_{k}}\right)$ is a basis of $\operatorname{span}_{\mathbb{R}}\left\{\left[X_{i}, X_{j}\right] \mid 1 \leq i, j \leq k\right\}$ for each $2 \leq k \leq d_{1}$.

(ii) If $2 \leq k \leq d_{1}, l_{k-1}<l_{k}$ and $l_{k-1}<j \leq l_{k}$, there is $i_{j} \in\{1, \ldots, k-1\}$ such that $\left[X_{i_{j}}, X_{k}\right]=Y_{j}$, and $l_{k-1}<j_{1}<j_{2} \leq l_{k}$ implies $i_{j_{1}}<i_{j_{2}}$.

We claim that for each $2 \leq k \leq d_{1}$ there exist a finite set $F_{k} \subseteq \mathbb{R}^{d_{1}} \times \mathbb{R}^{d_{2}}$ and a constant $\kappa_{k}>0$ such that the set

$$
\left\{(0, y) \in \mathbb{R}^{d_{1}} \times \mathbb{R}^{d_{2}}|| y_{j} \mid \leq \kappa_{k}, 1 \leq j \leq l_{k}, y_{j}=0, l_{k}+1 \leq j \leq d_{2}\right\}
$$

is contained in $\mathcal{C}\left(F_{k}\right)$. The theorem then follows from the claim in the case $k=d_{1}$ via Lemma 4.3.

Let $k=2, \kappa_{2}=1$. The sets

$$
S_{1}=\{(-2,0, \ldots, 0) *(0, \lambda, 0, \ldots, 0) \mid \lambda \in[-1,1]\}
$$

and

$$
S_{2}=\{(2,0, \ldots, 0) *(0, \lambda, 0, \ldots, 0) \mid \lambda \in[-1,1]\}
$$

are segments of integral curves of left-invariant, horizontal vector fields which are contained in the h-convex closure of

$$
F_{2}=\left\{g_{1}, g_{2}, g_{3}, g_{4}\right\}
$$

where

$$
\begin{array}{ll}
g_{1}=(-2,0, \ldots, 0) *(0,-1,0, \ldots, 0), & g_{2}=(-2,0, \ldots, 0) *(0,1,0, \ldots, 0), \\
g_{3}=(2,0, \ldots, 0) *(0,-1,0, \ldots, 0), & g_{4}=(2,0, \ldots, 0) *(0,1,0, \ldots, 0) .
\end{array}
$$

For each $y_{1} \in \mathbb{R}$ with $\left|y_{1}\right| \leq \kappa_{2}$,

$$
\begin{aligned}
S & =\left\{\left(0, \ldots, 0, y_{1}, 0, \ldots, 0\right) * \delta_{\lambda}\left(2, y_{1}, 0, \ldots, 0\right) \mid \lambda \in[-1,1]\right\} \\
& =\left\{\left(\lambda 2, \lambda y_{1}, 0, \ldots, 0, y_{1}, 0, \ldots, 0\right) \mid \lambda \in[-1,1]\right\}
\end{aligned}
$$

is a segment of an integral curve of a left-invariant, horizontal vector field, and the endpoints of $S$ are contained in $S_{1} \cup S_{2} \subseteq \mathcal{C}\left(F_{2}\right)$. Thus, $S \subseteq \mathcal{C}\left(F_{2}\right)$. Since $\left(0, \ldots, 0, y_{1}, 0, \ldots, 0\right)$ belongs to $S$, it follows that the set

$$
\left\{\left(0, \ldots, 0, y_{1}, 0, \ldots, 0\right)|| y_{1} \mid \leq \kappa_{2}\right\}
$$

is contained in $\mathcal{C}\left(F_{2}\right)$, which verifies the claim in the case $k=2$.

Let $2 \leq k<d_{1}$. Suppose that there exist a finite set $F_{k} \subseteq \mathbb{G}$ and a constant $\kappa_{k}>0$ such that the set

$$
\left\{(0, y) \in \mathbb{R}^{d_{1}} \times \mathbb{R}^{d_{2}}|| y_{j} \mid \leq \kappa_{k}, 1 \leq j \leq l_{k}, y_{j}=0, l_{k}+1 \leq j \leq d_{2}\right\}
$$

is contained in $\mathcal{C}\left(F_{k}\right)$. If $l_{k+1}=l_{k}$, the claim is also verified for $k+1$ and there is nothing to show. Assume therefore $\Delta=l_{k+1}-l_{k}>0$. By choice of $Y_{1}, \ldots, Y_{d_{2}}$, there exist $1 \leq i_{1}<$ $\ldots<i_{\Delta} \leq k$ such that $\left[X_{i_{j}}, X_{k+1}\right]=Y_{l_{k}+j}$ for $1 \leq j \leq \Delta$. In view of Lemma 4.3 , there exist a finite set $A_{k} \subseteq \mathbb{R}^{d_{1}} \times \mathbb{R}^{d_{2}}$ and a constant $0<K_{k} \leq \kappa_{k}$ such that the set $B$ consisting of the pairs $(x, y) \in \mathbb{R}^{d_{1}} \times \mathbb{R}^{d_{2}}$ with 
(i) $\left|x_{i}\right| \leq K_{k}$ for $i \in\left\{i_{1}, \ldots, i_{\Delta}\right\}$,

(ii) $x_{i}=0$ for $i \in\left\{1, \ldots, d_{1}\right\} \backslash\left\{i_{1}, \ldots, i_{\Delta}\right\}$,

(iii) $\left|y_{j}\right| \leq K_{k}$ for $1 \leq j \leq l_{k}$ and

(iv) $y_{j}=0$ for $l_{k}+1 \leq j \leq d_{2}$

is contained in $\mathcal{C}\left(A_{k}\right)$. Let $\kappa_{k+1}=K_{k}, g=\left(0, \ldots, 0, x_{k+1}, 0, \ldots, 0\right)$ with $x_{k+1}=2$ and define $F_{k+1}=l_{-g}\left(A_{k}\right) \cup l_{g}\left(A_{k}\right)$. Then $l_{-g}(B) \cup l_{g}(B)$ is contained in

$$
\begin{aligned}
l_{-g}\left(\mathcal{C}\left(A_{k}\right)\right) \cup l_{g}\left(\mathcal{C}\left(A_{k}\right)\right) & =\mathcal{C}\left(l_{-g}\left(A_{k}\right)\right) \cup \mathcal{C}\left(l_{g}\left(A_{k}\right)\right) \\
& \subseteq \mathcal{C}\left(l_{-g}\left(A_{k}\right) \cup l_{g}\left(A_{k}\right)\right)=\mathcal{C}\left(F_{k+1}\right) .
\end{aligned}
$$

Notice that the set $l_{-g}(B)$ consists of the pairs $(x, y) \in \mathbb{R}^{d_{1}} \times \mathbb{R}^{d_{2}}$ with

(i) $\left|x_{i}\right| \leq \kappa_{k+1}$ for $i \in\left\{i_{1}, \ldots, i_{\Delta}\right\}$,

(ii) $x_{k+1}=-2$,

(iii) $x_{i}=0$ for $i \in\left\{1, \ldots, d_{1}\right\} \backslash\left\{i_{1}, \ldots, i_{\Delta}, k+1\right\}$,

(iv) $\left|y_{j}\right| \leq \kappa_{k+1}$ for $1 \leq j \leq l_{k}$,

(v) $y_{j}=-x_{i_{j}}$ for $l_{k}+1 \leq j \leq l_{k+1}$ and

(vi) $y_{j}=0$ for $l_{k+1}+1 \leq j \leq d_{2}$.

Similarly, the set $l_{g}(B)$ consists of the pairs $(x, y) \in \mathbb{R}^{d_{1}} \times \mathbb{R}^{d_{2}}$ with

(i) $\left|x_{i}\right| \leq \kappa_{k+1}$ for $i \in\left\{i_{1}, \ldots, i_{\Delta}\right\}$,

(ii) $x_{k+1}=2$,

(iii) $x_{i}=0$ for $i \in\left\{1, \ldots, d_{1}\right\} \backslash\left\{i_{1}, \ldots, i_{\Delta}, k+1\right\}$,

(iv) $\left|y_{j}\right| \leq \kappa_{k+1}$ for $1 \leq j \leq l_{k}$,

(v) $y_{j}=x_{i j}$ for $l_{k}+1 \leq j \leq l_{k+1}$ and

(vi) $y_{j}=0$ for $l_{k+1}+1 \leq j \leq d_{2}$.

For $j=1, \ldots, l_{k+1}$, fix $y_{j} \in \mathbb{R}$ with $\left|y_{j}\right| \leq \kappa_{k+1}$. The set

$$
\begin{aligned}
S & =\left\{\left(0, \ldots, 0, y_{1}, \ldots, y_{l_{k+1}}, 0, \ldots, 0\right) * \delta_{\lambda}\left(x_{1}, \ldots, x_{d_{1}}, 0, \ldots, 0\right) \mid \lambda \in[-1,1]\right\} \\
& =\left\{\left(\lambda x_{1}, \ldots, \lambda x_{d_{1}}, y_{1}, \ldots, y_{l_{k+1}}, 0, \ldots, 0\right) \mid \lambda \in[-1,1]\right\},
\end{aligned}
$$

where $x_{i}=y_{j}$ if $i=i_{j}$ for some $l_{k}+1 \leq j \leq l_{k+1}, x_{k+1}=2$ and $x_{i}=0$ otherwise, is a segment of an integral curve of a left-invariant, horizontal vector field, and the endpoints of $S$ belong to $l_{-g}(B) \cup l_{g}(B)$. Thus, $S \subseteq \mathcal{C}\left(F_{k+1}\right)$. Since $\left(0, \ldots, 0, y_{1}, \ldots, y_{l_{k+1}}, 0, \ldots, 0\right)$ belongs to $S$, the set

$$
\left\{(0, y) \in \mathbb{R}^{d_{1}} \times \mathbb{R}^{d_{2}}|| y_{j} \mid \leq \kappa_{k+1}, 1 \leq j \leq l_{k+1}, y_{j}=0, l_{k+1}+1 \leq j \leq d_{2}\right\}
$$

is contained in $\mathcal{C}\left(F_{k+1}\right)$. This concludes the induction step and the proof.

\section{A Carnot group of step three which is not finitely h-convex}

Consider the Lie group $\mathbb{E} \equiv\left(\mathbb{R}^{4}, *\right)$, where

$$
\mathbb{R}^{4}=\left\{\left(x_{1}, x_{2}, y, z\right) \mid x_{1}, x_{2}, y, z \in \mathbb{R}\right\}
$$


and the product of $g=\left(x_{1}, x_{2}, y, z\right)$ and $g^{\prime}=\left(x_{1}^{\prime}, x_{2}^{\prime}, y^{\prime}, z^{\prime}\right)$ is defined to be

$$
g * g^{\prime}=\left(x_{1}+x_{1}^{\prime}, x_{2}+x_{2}^{\prime}, y+y^{\prime}, z+z^{\prime}\right)+P\left(g, g^{\prime}\right)
$$

with

$$
P\left(g, g^{\prime}\right)=\left(0,0, \frac{\left(x_{1} x_{2}^{\prime}-x_{2} x_{1}^{\prime}\right)}{2}, \frac{\left(x_{1} y^{\prime}-y x_{1}^{\prime}\right)}{2}+\frac{\left(x_{1}-x_{1}^{\prime}\right)\left(x_{1} x_{2}^{\prime}-x_{2} x_{1}^{\prime}\right)}{12}\right) .
$$

If $X_{1}, X_{2}, Y, Z$ denote the left-invariant vector fields uniquely determined by the conditions

$$
X_{1}(0)=\partial_{x_{1}}(0), X_{2}(0)=\partial_{x_{2}}(0), Y(0)=\partial_{y}(0), Z(0)=\partial_{z}(0),
$$

then the commutation relations

$$
\left[X_{1}, X_{2}\right]=Y,\left[X_{1}, Y\right]=Z,\left[X_{2}, Y\right]=\left[X_{1}, Z\right]=\left[X_{2}, Z\right]=[Y, Z]=0
$$

hold, and

$$
\operatorname{span}_{\mathbb{R}}\left\{X_{1}, X_{2}\right\} \oplus \operatorname{span}_{\mathbb{R}}\{Y\} \oplus \operatorname{span}_{\mathbb{R}}\{Z\}
$$

is a stratification of the Lie algebra $\mathfrak{e}$ of left-invariant vector fields on $\left(\mathbb{R}^{4}, *\right)$. Hence, $\mathbb{E}$ is a Carnot group of step 3 and of homogeneous dimension $Q=7 . \mathbb{E}$ is called the Engel group and $\mathfrak{e}$ the Engel algebra. Recall that

$$
\gamma: \mathbb{R} \rightarrow \mathbb{R}^{4}, \gamma(t)=g *\left(t x_{1}, t x_{2}, 0,0\right)
$$

is the integral curve of the left-invariant, horizontal vector field $X=x_{1} X_{1}+x_{2} X_{2}$ which passes through $g \in \mathbb{R}^{4}$ at time 0 .

Lemma 5.1. Let $\Gamma_{1}, \Gamma_{2}$ be integral curves of left-invariant, horizontal vector fields on $\left(\mathbb{R}^{4}, *\right)$. Define $M_{1}=(\bigcup \Gamma) \backslash \Gamma_{1}$, where the union is taken over all integral curves of left-invariant, horizontal vector fields which intersect $\Gamma_{1}$. If $\Gamma_{2}$ has more than two distinct intersections with $M_{1}$, then $\Gamma_{2}$ intersects $\Gamma_{1}$. Consequently, card $\left(\Gamma_{2} \cap\left(M_{1} \cup \Gamma_{1}\right)\right) \leq 2$ if $\Gamma_{1} \cap \Gamma_{2}=\emptyset$.

Proof. Notice first that by left translation, it suffices to prove the statement in the case where $\Gamma_{1}$ passes through 0 . We will only consider the case

$$
\Gamma_{1}=\{(\mu, \alpha \mu, 0,0) \mid \mu \in \mathbb{R}\}
$$

for some $\alpha \in \mathbb{R}$. The computations in the case

$$
\Gamma_{1}=\{(0, \mu, 0,0) \mid \mu \in \mathbb{R}\}
$$

are similar (but easier). We have

$$
\begin{aligned}
& (\mu, \alpha \mu, 0,0) *\left(x_{1}, x_{2}, 0,0\right) \\
& \quad=\left(\mu+x_{1}, \alpha \mu+x_{2}, \frac{\mu\left(x_{2}-\alpha x_{1}\right)}{2}, \frac{\left(\mu-x_{1}\right) \mu\left(x_{2}-\alpha x_{1}\right)}{12}\right) \\
& \quad=(u, v, w, f(u, v, w)),
\end{aligned}
$$

with $u=\mu+x_{1}, v=\alpha \mu+x_{2}, w=\mu\left(x_{2}-\alpha x_{1}\right) / 2$. A computation gives

$$
M_{1}=\left\{\left(u, v, w, \frac{w}{6}\left(\frac{4 w}{v-\alpha u}-u\right)\right) \mid u, v, w \in \mathbb{R}, v-\alpha u \neq 0\right\} .
$$


Let $\left(x_{1}, x_{2}, y, z\right) \in \mathbb{R}^{4}$ and suppose that $\Gamma_{2}$ passes through $\left(x_{1}, x_{2}, y, z\right)$. As above, we will only consider the case $\Gamma_{2}=\left\{\left(x_{1}, x_{2}, y, z\right) *(\lambda, \beta \lambda, 0,0) \mid \lambda \in \mathbb{R}\right\}$ for some $\beta \in \mathbb{R}$. The computations in the case $\Gamma_{2}=\left\{\left(x_{1}, x_{2}, y, z\right) *(0, \lambda, 0,0) \mid \lambda \in \mathbb{R}\right\}$ are again similar and easier. We have

$$
\Gamma_{2}=\left\{\left(x_{1}, x_{2}, y, z\right) *(\lambda, \beta \lambda, 0,0) \mid \lambda \in \mathbb{R}\right\}
$$

with

$$
\begin{aligned}
& \left(x_{1}, x_{2}, y, z\right) *(\lambda, \beta \lambda, 0,0) \\
& \quad=\left(x_{1}+\lambda, x_{2}+\beta \lambda, y+\frac{\lambda\left(x_{1} \beta-x_{2}\right)}{2}, z+\frac{(-y) \lambda}{2}+\frac{\left(x_{1}-\lambda\right) \lambda\left(x_{1} \beta-x_{2}\right)}{12}\right) .
\end{aligned}
$$

Suppose first that $x_{1} \beta-x_{2}=0$. By hypothesis,

$$
\frac{y}{6}\left(\frac{4 y}{x_{2}+\beta \lambda-\alpha x_{1}-\alpha \lambda}-\left(x_{1}+\lambda\right)\right)=z+\frac{(-y) \lambda}{2}
$$

holds for at least three distinct values of $\lambda$. After simplification of this expression, we obtain $a_{2} \lambda^{2}+a_{1} \lambda+a_{0}=0$ for at least three distinct values of $\lambda$, where

$$
\begin{aligned}
& a_{0}=4 y^{2}+\left(\alpha x_{1}-x_{2}\right)\left(x_{1} y+6 z\right) \\
& a_{1}=2 y\left(x_{2}-\alpha x_{1}\right)+(\alpha-\beta)\left(x_{1} y+6 z\right) \\
& a_{2}=2 y(\beta-\alpha) \lambda^{2} .
\end{aligned}
$$

If $\alpha-\beta=0$, then $\alpha x_{1}-x_{2}=0, y=0$, and thus

$$
\Gamma_{2}=\left\{\left(x_{1}+\lambda, \alpha x_{1}+\alpha \lambda, 0, z\right) \mid \lambda \in \mathbb{R}\right\}=\Gamma_{1} .
$$

Hence, $\Gamma_{2} \cap M_{1}=\emptyset$, a contradiction. This forces $\alpha-\beta \neq 0$, which implies $y=z=0$ and

$$
\Gamma_{2}=\left\{\left(x_{1}, \beta x_{1}, 0,0\right) *(\lambda, \beta \lambda, 0,0) \mid \lambda \in \mathbb{R}\right\}=\left\{\left(x_{1}+\lambda, \beta\left(x_{1}+\lambda\right), 0,0\right) \mid \lambda \in \mathbb{R}\right\} .
$$

Thus, $0 \in \Gamma_{1} \cap \Gamma_{2}$, and the claim follows.

Suppose now that $x_{1} \beta-x_{2} \neq 0$. Then $\Gamma_{2}$ intersects the hyperplane

$$
\left\{\left(x_{1}^{\prime}, x_{2}^{\prime}, y^{\prime}, z^{\prime}\right) \in \mathbb{R}^{4} \mid y^{\prime}=0\right\}
$$

at some point $\left(x_{1}^{\prime}, x_{2}^{\prime}, 0, z^{\prime}\right)$, and we can write

$$
\begin{aligned}
\Gamma_{2} & =\left\{\left(x_{1}^{\prime}, x_{2}^{\prime}, 0, z^{\prime}\right) *(\lambda, \beta \lambda, 0,0) \mid \lambda \in \mathbb{R}\right\} \\
& =\left\{\left(x_{1}^{\prime}+\lambda, x_{2}^{\prime}+\beta \lambda, \frac{\lambda\left(x_{1}^{\prime} \beta-x_{2}^{\prime}\right)}{2}, z^{\prime}+\frac{\left(x_{1}^{\prime}-\lambda\right) \lambda\left(x_{1}^{\prime} \beta-x_{2}^{\prime}\right)}{12}\right) \mid \lambda \in \mathbb{R}\right\}
\end{aligned}
$$

with $x_{1}^{\prime} \beta-x_{2}^{\prime} \neq 0$. By hypothesis,

$$
\frac{\lambda\left(x_{1}^{\prime} \beta-x_{2}^{\prime}\right)}{12}\left(\frac{2 \lambda\left(x_{1}^{\prime} \beta-x_{2}^{\prime}\right)}{x_{2}^{\prime}+\beta \lambda-\alpha x_{1}^{\prime}-\alpha \lambda}-\left(x_{1}^{\prime}+\lambda\right)\right)=z^{\prime}+\frac{\left(x_{1}^{\prime}-\lambda\right) \lambda\left(x_{1}^{\prime} \beta-x_{2}^{\prime}\right)}{12},
$$

holds for at least three distinct values of $\lambda$. After simplification of this expression, we obtain $a_{2} \lambda^{2}+a_{1} \lambda+a_{0}=0$ for at least three distinct values of $\lambda$, where

$$
\begin{aligned}
& a_{0}=12 z^{\prime}\left(x_{2}^{\prime}-\alpha x_{1}^{\prime}\right) \\
& a_{1}=2\left(x_{1}^{\prime} \beta-x_{2}^{\prime}\right) x_{1}^{\prime}\left(\alpha x_{1}^{\prime}-x_{2}^{\prime}\right)+12 z^{\prime}(\alpha-\beta) \\
& a_{2}=2\left(x_{1}^{\prime} \beta-x_{2}^{\prime}\right)^{2}+2\left(x_{1}^{\prime} \beta-x_{2}^{\prime}\right) x_{1}^{\prime}(\alpha-\beta) .
\end{aligned}
$$


If $z^{\prime} \neq 0$, then $x_{2}^{\prime}-\alpha x_{1}^{\prime}=\beta-\alpha=0$, and thus

$$
\Gamma_{2}=\left\{\left(x_{1}^{\prime}+\lambda, \alpha x_{1}^{\prime}+\alpha \lambda, 0, z^{\prime}\right) \mid \lambda \in \mathbb{R}\right\}=\Gamma_{1} .
$$

Hence, $\Gamma_{2} \cap M_{1}=\emptyset$, a contradiction. This forces $z^{\prime}=0$ and thus $\left(x_{1}^{\prime} \beta-x_{2}^{\prime}\right) x_{1}^{\prime}\left(\alpha x_{1}^{\prime}-x_{2}^{\prime}\right)=0$. If $x_{1}^{\prime}=0$, then $x_{2}^{\prime}=0$, whence $x_{1}^{\prime} \beta-x_{2}^{\prime}=0$. This contradiction forces $\alpha x_{1}^{\prime}-x_{2}^{\prime}=0$, which implies

$$
\Gamma_{2}=\left\{\left(x_{1}^{\prime}, \alpha x_{1}^{\prime}, 0,0\right) *(\lambda, \beta \lambda, 0,0) \mid \lambda \in \mathbb{R}\right\} .
$$

Thus, $\left(x_{1}^{\prime}, \alpha x_{1}^{\prime}, 0,0\right) \in \Gamma_{1} \cap \Gamma_{2}$, and the claim follows.

Lemma 5.2. Let $S_{1}$ and $S_{2}$ be bounded, closed, intersecting segments (possibly points) of distinct integral curves $\Gamma_{1}, \Gamma_{2}$ of left-invariant, horizontal vector fields. Let $S$ be a bounded, closed segment of an integral curve of a left-invariant, horizontal vector field, and suppose that one endpoint $g_{1}$ of $S$ belongs to $S_{1}$ and the other endpoint $g_{2}$ belongs to $S_{2}$. Then $S \subseteq S_{1}$ or $S \subseteq S_{2}$.

Proof. After a left translation, we can assume that

$$
0 \in S_{1} \cap S_{2}, \quad \Gamma_{1}=\left\{\left(\lambda x_{1}, \lambda x_{2}, 0,0\right) \mid \lambda \in \mathbb{R}\right\} \quad \text { and } \quad \Gamma_{2}=\left\{\left(\lambda x_{1}^{\prime}, \lambda x_{2}^{\prime}, 0,0\right) \mid \lambda \in \mathbb{R}\right\}
$$

for suitable, linearly independent $\left(x_{1}, x_{2}\right),\left(x_{1}^{\prime}, x_{2}^{\prime}\right) \in \mathbb{R}^{2}$ with $\left(x_{1}^{2}+x_{2}^{2}\right)=\left(x_{1^{\prime}}^{2}+x_{2^{\prime}}^{2}\right)=$ 1. We have

$$
g_{1}=\left(\lambda x_{1}, \lambda x_{2}, 0,0\right)
$$

for some $\lambda \in \mathbb{R}$ and

$$
\begin{aligned}
g_{2} & =g_{1} *\left(u_{1}, u_{2}, 0,0\right)=\left(\lambda x_{1}, \lambda x_{2}, 0,0\right) *\left(u_{1}, u_{2}, 0,0\right) \\
& =\left(\lambda x_{1}+u_{1}, \lambda x_{2}+u_{2}, \frac{\lambda\left(x_{1} u_{2}-x_{2} u_{1}\right)}{2}, \frac{\left(\lambda x_{1}-u_{1}\right) \lambda\left(x_{1} u_{2}-x_{2} u_{1}\right)}{12}\right)
\end{aligned}
$$

for some $\left(u_{1}, u_{2}\right) \in \mathbb{R}^{2} . g_{2} \in \Gamma_{2}$ implies that $\lambda=0$ or that $\left(x_{1}, x_{2}\right)$ and $\left(u_{1}, u_{2}\right)$ are linearly dependent. If $\lambda=0$, then $g_{1}=0 \in S_{2}$. If $\left(x_{1}, x_{2}\right)$ and $\left(u_{1}, u_{2}\right)$ are linearly dependent, then $g_{2} \in \Gamma_{1}$, forcing $g_{2}=0 \in S_{1}$ since $\Gamma_{1} \cap \Gamma_{2}=\{0\}$.

Lemma 5.3. If $A \subseteq \mathbb{E}$ is a finite union of bounded, closed segments $S_{1}, \ldots, S_{k}$ (possibly points) of integral curves $\Gamma_{1}, \ldots, \Gamma_{k}$ of left-invariant, horizontal vector fields, then $\mathrm{H}(A)$ is contained in a finite union of bounded, closed segments (possibly points) of integral curves of left-invariant, horizontal vector fields.

Proof. Enlarging $A$ if necessary, we can assume that

(i) $\Gamma_{1}, \ldots, \Gamma_{k}$ are all distinct and

(ii) $\Gamma_{i} \cap \Gamma_{j} \neq \emptyset$ for some $i, j \in\{1, \ldots, k\}$ implies $S_{i} \cap S_{j} \neq \emptyset$.

Note that $\mathrm{H}(A)$ is the union of all bounded, closed segments of integral curves of left-invariant, horizontal vector fields with endpoints in $A$. Hence, a bounded, closed segment $S$ of an integral curve of some left-invariant, horizontal vector field is contained in $\mathrm{H}(A)$ if and only if there exist $i, j \in\{1, \ldots, k\}$ such that one endpoint $g_{i}$ of $S$ belongs to $S_{i}$ and the other endpoint $g_{j}$ belongs to $S_{j}$. We can assume $i \neq j$, for otherwise $S \subseteq S_{i}=S_{j}$ since $S$ is determined by its endpoints. If $\Gamma_{i} \cap \Gamma_{j} \neq \emptyset$, then $S_{i} \cap S_{j} \neq \emptyset$ and $S \subseteq S_{i}$ or $S \subseteq S_{j}$ by Lemma 5.2. If $\Gamma_{i} \cap \Gamma_{j}=\emptyset$, then $S$ 
is one out of four (at most) possible segments. Indeed, by virtue of Lemma 5.1, $g_{i}$ is one out of two (at most) possible intersection points of $\Gamma_{i}$ with $M_{j}=(\bigcup \Gamma) \backslash \Gamma_{j}$. Similarly, $g_{j}$ is one out of two (at most) possible intersection points of $\Gamma_{j}$ with $M_{i}=(\bigcup \Gamma) \backslash \Gamma_{i}$. Since $S$ is determined by its endpoints, the claim follows.

In view of $\mathcal{C}(A)=\mathrm{H}^{\infty}(A)=\bigcup_{k \in \mathbb{N}} \mathrm{H}^{k}(A)$, the following theorem is an immediate consequence of Lemma 5.3.

Theorem 5.4. If $F \subseteq \mathbb{E}$ is finite, then the $h$-convex closure $\mathcal{C}(F)$ of $F$ is contained in a countable union of bounded, closed segments (possibly points) of integral curves of left-invariant, horizontal vector fields.

\section{Local Lipschitz continuity of measurable h-convex functions}

\subsection{Density upper bound at the boundary of measurable h-convex sets}

This subsection is devoted to the proof of Theorem 1.3.

We say that a smooth manifold $M^{m} \subseteq \mathbb{R}^{d}$ is a submanifold if the manifold topology is the topology induced by $\mathbb{R}^{d}$. The superscript $m$ denotes the topological dimension of the manifold $(1 \leq m \leq d)$. If $M^{m}$ is a smooth submanifold and $X_{1}, \ldots, X_{n}$ are smooth vector fields on $\mathbb{R}^{d}$, then the singular set or characteristic set of $M^{m}$ with respect to the vector fields $X_{1}, \ldots, X_{n}$ is the set

$$
\Sigma\left(M^{m}\right)=\left\{p \in M^{m} \mid X_{i}(p) \in \mathrm{T}_{p} M^{m}, i=1, \ldots, n\right\} .
$$

Characteristic points have been extensively studied because of their fundamental importance in several problems of geometry and analysis related to systems of vector fields satisfying Hörmander's condition. In this article, we only need the most basic estimate of the size of the characteristic set, namely: If $X_{1}, \ldots, X_{n}$ and their commutators of order at most $s$ span $\mathrm{T}_{x} \mathbb{R}^{d}$ at each $x \in \mathbb{R}^{d}$, then $\mathcal{H}_{E}^{m}\left(\Sigma\left(M^{m}\right)\right)=0$. Here $\mathcal{H}_{E}^{m}$ denotes $m$-dimensional Hausdorff measure with respect to the Euclidean metric on $\mathbb{R}^{d}$. This estimate was proved by Derridj (cf. [7]) for characteristic sets of smooth submanifolds of codimension 1.

Lemma 6.1. Let $Y=\sum_{i=1}^{d} a_{i} \partial_{i}, Z=\sum_{j=1}^{d} b_{j} \partial_{j}$ be smooth vector fields on $\mathbb{R}^{d}$. Let $1 \leq m<d, \mathbb{R}^{m}=\left\{x \in \mathbb{R}^{d} \mid x_{v}=0, m+1 \leq v \leq d\right\},[Y, Z]=\sum_{k=1}^{d} c_{k} \partial_{k}$. Then the set $\Sigma$ consisting of the points $x \in \mathbb{R}^{m}$ such that $a_{i}(x)=b_{j}(x)=0$ for all $m<i, j \leq d$ and $c_{k}(x) \neq 0$ for some $m<k \leq d$ has vanishing $\mathcal{H}_{E}^{m}$ measure.

Proof. We compute

$$
\begin{aligned}
{[Y, Z] } & =\sum_{i=1}^{d} \sum_{j=1}^{d} a_{i} \partial_{i}\left(b_{j} \partial_{j}\right)-b_{j} \partial_{j}\left(a_{i} \partial_{i}\right)=\sum_{i=1}^{d} \sum_{j=1}^{d} a_{i}\left(\partial_{i} b_{j}\right) \partial_{j}-b_{j}\left(\partial_{j} a_{i}\right) \partial_{i} \\
& =\sum_{k=1}^{d}\left(\sum_{l=1}^{d} a_{l}\left(\partial_{l} b_{k}\right)-b_{l}\left(\partial_{l} a_{k}\right)\right) \partial_{k}
\end{aligned}
$$

Thus, $c_{k}=\sum_{l=1}^{d} a_{l}\left(\partial_{l} b_{k}\right)-b_{l}\left(\partial_{l} a_{k}\right)$. For $m<k \leq d, 1 \leq l \leq m$, let us consider the sets

$$
A_{k, l}=\left\{x \in \mathbb{R}^{m} \mid a_{k}(x)=0, \partial_{l} a_{k}(x) \neq 0\right\}
$$


and

$$
B_{k, l}=\left\{x \in \mathbb{R}^{m} \mid b_{k}(x)=0, \partial_{l} b_{k}(x) \neq 0\right\} .
$$

Since $\Sigma \subseteq \bigcup_{m<k \leq d, 1 \leq l \leq m}\left(A_{k, l} \cup B_{k, l}\right)$, it suffices to show that $\mathcal{H}_{E}^{m}\left(A_{k, l}\right)$ and $\mathcal{H}_{E}^{m}\left(B_{k, l}\right)$ vanish for $m<k \leq d$ and $1 \leq l \leq m$. Let us prove $\mathcal{H}_{E}^{m}\left(A_{d, m}\right)=0$ for instance. Consider $\mathbb{R}^{m-1}=$ $\left\{x \in \mathbb{R}^{d} \mid x_{v}=0, m \leq v \leq d\right\}$. By Fubini's theorem,

$$
\mathcal{H}_{E}^{m}\left(A_{d, m}\right)=\int_{\mathbb{R}^{m-1}} \mathcal{H}_{E}^{1}\left(\left\{x+t e_{m} \mid t \in \mathbb{R}\right\} \cap A_{d, m}\right) d \mathcal{H}_{E}^{m-1}(x)
$$

Fix $x \in \mathbb{R}^{m-1}$. The set

$$
\left\{t \in \mathbb{R} \mid a_{d}\left(x+t e_{m}\right)=0, \partial_{m} a_{d}\left(x+t e_{m}\right) \neq 0\right\}
$$

consists of isolated points. Therefore $\mathcal{H}_{E}^{1}\left(\left\{x+t e_{m} \mid t \in \mathbb{R}\right\} \cap A_{d, m}\right)=0$, whence $\mathcal{H}_{E}^{m}\left(A_{d, m}\right)=0$.

Given smooth vector fields $X_{1}, \ldots, X_{n}$ in $\mathbb{R}^{d}$ and a multiindex $I$ of length $|I|=\ell$, i.e., $I \in\{1, \ldots, n\}^{\ell}$, we let $X_{I}=X_{i}$ if $\ell=1$ and $I=(i), X_{I}=\left[X_{\left(i_{1}, \ldots, i_{\ell-1}\right)}, X_{i_{\ell}}\right]$ if $\ell \geq 2$ and $I=\left(i_{1}, \ldots, i_{\ell}\right)$, and we write $X_{I}=\sum_{j=1}^{d} a_{I, j} \partial_{j}$.

Lemma 6.2. Let $X_{1}, \ldots, X_{n}$ be smooth vector fields on $\mathbb{R}^{d}$. Fix $1 \leq m<d$ and consider $\mathbb{R}^{m}=\left\{x \in \mathbb{R}^{d} \mid x_{v}=0, m+1 \leq v \leq d\right\}$. The set $\Sigma$ consisting of the points $x \in \mathbb{R}^{m}$ such that

(i) $a_{(i), j}(x)=0$ for all $i=1, \ldots, n$ and $m<j \leq d$ and

(ii) $a_{I, j}(x) \neq 0$ for some $I$ with $|I| \leq s$ and some $m<j \leq d$

has vanishing $\mathcal{H}_{E}^{m}$ measure for all $s \in \mathbb{N}$.

Proof. Let $s \in \mathbb{N}, s \geq 2$. For $\ell=1, \ldots, s-1$, let $\Sigma_{\ell}$ be the set of points $x \in \mathbb{R}^{m}$ such that

(i) $a_{I, j}(x)=0$ for all $I$ with $|I| \leq \ell$ and all $m<j \leq d$ and

(ii) $a_{I_{0}, j}(x) \neq 0$ for some $I_{0}$ with $\left|I_{0}\right|=\ell+1$ and some $m<j \leq d$.

Clearly $\Sigma=\bigcup_{\ell=1}^{s-1} \Sigma_{\ell}$. Hence, it is enough to show $\mathcal{H}_{E}^{m}\left(\Sigma_{\ell}\right)=0$ for $1 \leq \ell \leq s-1$. Note that

$$
\Sigma_{\ell} \subseteq \bigcup_{|I|=\ell, 1 \leq i \leq n} \Sigma_{I,(i)},
$$

where $\Sigma_{I,(i)}$ is the set of points $x \in \mathbb{R}^{m}$ such that

(i) $a_{I, j}(x)=a_{i, j}(x)=0$ for $m<j \leq d$ and

(ii) $a_{(I, i), j}(x) \neq 0$ for some $m<j \leq d$.

We have $\mathcal{H}_{E}^{m}\left(\Sigma_{I,(i)}\right)=0$ by Lemma 6.1 , concluding the proof.

Theorem 6.3. Let $M^{m} \subseteq \mathbb{R}^{d}$ be a smooth submanifold. Let $X_{1}, \ldots, X_{n}$ be smooth vector fields on $\mathbb{R}^{d}$ such that the subspace of $\mathrm{T}_{x} \mathbb{R}^{d}$ spanned by the commutators of order at most $s$ has dimension $d$ at each $x \in \mathbb{R}^{d}$. Then $\mathcal{H}_{E}^{m}\left(\Sigma\left(M^{m}\right)\right)=0$. 
Proof. It suffices to show that for each $p \in M^{m}$, there exists an open neighborhood $U$ of $p$ in $M^{m}$ such that $\mathcal{H}_{E}^{m}\left(\Sigma\left(M^{m}\right) \cap U\right)=0$. Fix $p \in M^{m}$. Let $V$ be an open neighborhood of $p$ in $\mathbb{R}^{d}$ and let $\varphi: V \rightarrow \mathbb{R}^{d}$ be a diffeomorphism such that $\varphi\left(M^{m} \cap V\right)=\mathbb{R}^{m}=\left\{x \in \mathbb{R}^{d} \mid x_{v}=0, m<\right.$ $v \leq d\}$. Let us write $\tilde{X}_{i}=d \varphi\left(X_{i}\right)$ and let us observe that for each multiindex $I \in\{1, \ldots, d\}^{\ell}$ of length $|I|=\ell$, we have $\tilde{X}_{I}=d \varphi\left(X_{I}\right)$. In particular,

$$
\operatorname{span}_{\mathbb{R}}\left\{\widetilde{X}_{I}(x)|| I \mid \leq s\right\}=\mathrm{T}_{x} \mathbb{R}^{d}
$$

at each $x \in \mathbb{R}^{d}$. Thus, if $x \in \varphi\left(\Sigma\left(M^{m}\right) \cap V\right)$, then $\widetilde{X}_{i}(x) \in \mathrm{T}_{x} \mathbb{R}^{m}, i=1, \ldots, n$, and $\widetilde{X}_{I}(x) \notin \mathrm{T}_{x} \mathbb{R}^{m}$ for some $I$ with $|I| \leq s$. We have $\mathcal{H}_{E}^{m}\left(\varphi\left(\Sigma\left(M^{m}\right) \cap V\right)\right)=0$ by Lemma 6.2 , and consequently, $\mathcal{H}_{E}^{m}\left(\Sigma\left(M^{m}\right) \cap V\right)=0$.

Lemma 6.4. Let $\mathbb{G} \equiv\left(\mathbb{R}^{d}, *\right)$ be a Carnot group, $C \subseteq \mathbb{R}^{d}$ an h-convex subset such that $0 \in \mathbb{R}^{d} \backslash C$. Then there exist smooth submanifolds $M^{1}, M^{2}, \ldots, M^{d}$ in $\mathbb{R}^{d}$ such that

(i) $M^{m} \subseteq B(0, m / d)$,

(ii) $\mathcal{H}_{E}^{m}\left(M^{m}\right) \leq b_{m}<+\infty$ and

(iii) $\mathcal{H}_{E}^{m}\left(M^{m} \backslash C\right) \geq c_{m}>0$

for $m=1, \ldots, d . M^{m}, b_{m}$ and $c_{m}$ are independent of $C$.

Proof. Let $\left(X_{1}, \ldots, X_{d_{1}}\right)$ be a basis of the first layer in the given stratification of the Lie algebra of left-invariant vector fields on $\left(\mathbb{R}^{d}, *\right)$. Given $x_{0} \in \mathbb{R}^{d} \backslash C$ and $1 \leq j \leq d_{1}$, let $\gamma_{j, x_{0}}: \mathbb{R} \rightarrow \mathbb{R}^{d}$ denote the integral curve of the vector field $X_{j}$ which passes through $x_{0}$ at time 0 . By definition of h-convexity,

$$
\gamma_{j, x_{0}}((-\infty, 0]) \cap C=\emptyset \quad \text { or } \quad \gamma_{j, x_{0}}([0,+\infty)) \cap C=\emptyset .
$$

Choose $\eta>0$ such that $\gamma_{1,0}((-\eta, \eta)) \subseteq B(0,1 / d)$. By $(6.1)$, we have

$$
\gamma_{1,0}((-\eta, 0]) \cap C=\emptyset \quad \text { or } \quad \gamma_{1,0}([0, \eta)) \cap C=\emptyset .
$$

We let $M^{1}=\gamma_{1,0}((-\eta, \eta)), b_{1}=\mathcal{H}_{E}^{1}\left(M^{1}\right)$ and

$$
c_{1}=\min \left\{\mathcal{H}_{E}^{1}\left(\gamma_{1,0}((-\eta, 0))\right), \mathcal{H}_{E}^{1}\left(\gamma_{1,0}((0, \eta))\right)\right\} .
$$

Clearly $M_{1}, b_{1}$ and $c_{1}$ have the required properties.

Let $1 \leq m<d$. Suppose we had already constructed smooth submanifolds $M^{1}, M^{2}, \ldots, M^{m}$ which satisfy our claims. Define $A^{m}=M^{m} \backslash C$. For $1 \leq j \leq d_{1}, k \in \mathbb{N}$, denote by $F_{j, k}^{m}$ the closed set consisting of points $p \in M^{m}$ such that

$$
\max \left\{\left(\frac{X_{j}(p)}{\left(X_{j}(p), X_{j}(p)\right)^{1 / 2}}, Y(p)\right) \mid Y(p) \in\left(\mathrm{T}_{p} M^{m}\right)^{\perp},(Y(p), Y(p))=1\right\} \leq \frac{1}{k} .
$$

Let $F_{k}^{m}=\bigcap_{j=1}^{d_{1}} F_{j, k}^{m}$. By Theorem 6.3, we have

$$
\lim _{k \rightarrow+\infty} \mathcal{H}_{E}^{m}\left(F_{k}^{m}\right)=\mathcal{H}_{E}^{m}\left(\bigcap_{k \in \mathbb{N}} F_{k}^{m}\right)=\mathcal{H}_{E}^{m}\left(\Sigma\left(M^{m}\right)\right)=0
$$

Let

$$
q_{m}=\frac{1}{2}\left(\frac{b_{m}}{b_{m}+c_{m} / d_{1}}+1\right)
$$


Then

$$
\frac{\mathcal{H}_{E}^{m}\left(M^{m}\right)}{\mathcal{H}_{E}^{m}\left(M^{m}\right)+c_{m} / d_{1}}<q_{m}<1 .
$$

There is a least $k \in \mathbb{N}$ such that $\mathcal{H}_{E}^{m}\left(F_{k}^{m}\right) \leq\left(1-q_{m}\right) c_{m}$. This implies that $\mathcal{H}_{E}^{m}\left(A^{m} \backslash F_{k}^{m}\right) \geq$ $q_{m} c_{m}$, whence

$$
\mathcal{H}_{E}^{m}\left(A^{m} \backslash F_{j, k}^{m}\right) \geq q_{m} c_{m} / d_{1}
$$

for some $1 \leq j \leq d_{1}$. There exists $\Omega_{j}^{m} \Subset M^{m} \backslash F_{j, k}^{m}$, such that

$$
\mathcal{H}_{E}^{m}\left(\Omega_{j}^{m}\right) \geq q_{m} \mathcal{H}_{E}^{m}\left(M^{m} \backslash F_{j, k}^{m}\right)
$$

Hence,

$$
\mathcal{H}_{E}^{m}\left(\left(M^{m} \backslash F_{j, k}^{m}\right) \backslash \Omega_{j}^{m}\right) \leq\left(1-q_{m}\right) \mathcal{H}_{E}^{m}\left(M^{m} \backslash F_{j, k}^{m}\right)
$$

It follows that

$$
\mathcal{H}_{E}^{m}\left(A^{m} \cap \Omega_{j}^{m}\right) \geq \mathcal{H}_{E}^{m}\left(A^{m} \backslash F_{j, k}^{m}\right)-\left(1-q_{m}\right) \mathcal{H}_{E}^{m}\left(M^{m} \backslash F_{j, k}^{m}\right) .
$$

Given $p \in \Omega_{j}^{m}$, let $\gamma_{j, p}: \mathbb{R} \rightarrow \mathbb{R}^{d}$ be the integral curve of $X_{j}$ which passes through $p$ at time 0 . When $\epsilon_{j}^{m}>0$ is sufficiently small, the smooth mapping

$$
\Phi_{j}^{m}: \Omega_{j}^{m} \times\left(-\epsilon_{j}^{m}, \epsilon_{j}^{m}\right) \rightarrow \mathbb{R}^{d}, \quad \Phi_{j}^{m}(p, t)=\gamma_{j, p}(t)
$$

is bi-Lipschitz for some constant $0<L_{j}^{m}<+\infty$ and

$$
M_{j}^{m+1}=\Phi_{j}^{m}\left(\Omega_{j}^{m} \times\left(-\epsilon_{j}^{m}, \epsilon_{j}^{m}\right)\right)
$$

is a smooth submanifold.

With the help of $\Phi_{j}^{m}$, using (6.1) and the estimate

$$
\mathcal{H}_{E}^{m+1}(A \times I) \geq \frac{\alpha(m+1)}{\alpha(m) \alpha(1)} \mathcal{H}_{E}^{m}(A) \mathcal{H}_{E}^{1}(I)
$$

(see [9, 2.10.27]), valid if $I \subseteq \mathbb{R}$ is an interval and $A \subseteq \mathbb{R}^{m}$ is an arbitrary subset, it follows that there exists a constant $\lambda_{j}^{m}>0$ such that

$$
\mathcal{H}_{E}^{m+1}\left(\Phi_{j}^{m}\left(\Omega_{j}^{m} \times\left(-\epsilon_{j}^{m}, \epsilon_{j}^{m}\right)\right) \backslash C\right) \geq \lambda_{j}^{m} \mathcal{H}_{E}^{m}\left(A^{m} \cap \Omega_{j}^{m}\right)
$$

Combining (6.4) and (6.5), we obtain

$$
\mathcal{H}_{E}^{m+1}\left(M_{j}^{m+1} \backslash C\right) \geq \lambda_{j}^{m}\left(\mathcal{H}_{E}^{m}\left(A^{m} \backslash F_{j, k}^{m}\right)-\left(1-q_{m}\right) \mathcal{H}_{E}^{m}\left(M^{m} \backslash F_{j, k}^{m}\right)\right) .
$$

Let $M^{m+1}=M_{j}^{m+1}$. Then, from (6.2) and (6.3), we get

$$
\mathcal{H}_{E}^{m+1}\left(M^{m+1} \backslash C\right) \geq \lambda_{j}^{m}\left(q_{m} c_{m} / d_{1}-\left(1-q_{m}\right) \mathcal{H}_{E}^{m}\left(M^{m}\right)\right) \geq c_{m+1}>0
$$


Proof of Theorem 1.3. As usual, we assume (as we may) $\mathbb{G} \equiv\left(\mathbb{R}^{d}, *\right)$. We have to show that there exists $0 \leq c<1$ such that

$$
\frac{\mathcal{H}^{Q}\left(B\left(x_{0}, r\right) \cap C\right)}{\mathcal{H}^{Q}\left(B\left(x_{0}, r\right)\right)} \leq c
$$

for all $0<r<+\infty$, whenever $C \subseteq\left(\mathbb{R}^{d}, *\right)$ is an h-convex, measurable subset and $x_{0} \in \partial C$ is a point on its boundary.

Fix $1<\lambda<\left(\mathcal{H}_{E}^{d}(B(0,1)) /\left(\mathcal{H}_{E}^{d}(B(0,1))-c_{d}\right)\right)^{1 / Q}$, where $c_{d}$ is the constant appearing in Lemma 6.4. Pick $x \in \mathbb{R}^{d} \backslash C$ sufficiently close to $x_{0}$, in such a way that $B\left(x_{0}, r\right) \subseteq B(x, \lambda r)$. The set $\widetilde{C}=\delta_{1 /(\lambda r)} \circ l_{-x}(C)$ is h-convex, measurable and does not contain 0. By Lemma 6.4, there exists a smooth submanifold $M^{d} \subseteq B(0,1)$, such that $\mathcal{H}_{E}^{d}\left(M^{d} \backslash \widetilde{C}\right) \geq c_{d}$. Thus,

$$
\begin{aligned}
\frac{\mathcal{H}^{Q}\left(B\left(x_{0}, r\right) \cap C\right)}{\mathcal{H}^{Q}\left(B\left(x_{0}, r\right)\right)} & \leq \frac{\mathcal{H}^{Q}\left(l_{x} \circ \delta_{\lambda r}(B(0,1) \cap \widetilde{C})\right)}{\mathcal{H}^{Q}\left(l_{x_{0}} \circ \delta_{r}(B(0,1))\right)}=\frac{\lambda^{Q} \mathcal{H}^{Q}(B(0,1) \cap \widetilde{C})}{\mathcal{H}^{Q}(B(0,1))} \\
& =\lambda^{Q}-\lambda^{Q} \frac{\mathcal{H}_{E}^{d}(B(0,1) \backslash \widetilde{C})}{\mathcal{H}_{E}^{d}(B(0,1))}
\end{aligned}
$$

and the claim follows with $c=\lambda^{Q}\left(1-c_{d} / \mathcal{H}_{E}^{d}(B(0,1))\right)$.

\subsection{Local Lipschitz continuity of measurable h-convex functions}

In this subsection, we use Theorem 1.3 to prove the following more general version of Theorem 1.4.

Theorem 6.5. Let $\mathbb{G}$ be a Carnot group, $\Omega \subseteq \mathbb{G}$ an $h$-convex, open subset and $u: \Omega \rightarrow \mathbb{R}$ an $h$-convex function. Suppose there exists a sequence $\left\{b_{k}\right\}_{k \in \mathbb{N}}$ of real numbers such that $b_{k} \rightarrow+\infty$ and $\left\{g \in \Omega \mid u(g)<b_{k}\right\}$ is measurable for all $k \in \mathbb{N}$. Then $u$ is locally Lipschitz continuous with respect to any intrinsic metric on $\mathbb{G}$.

Proof. Let $\mathbb{G}, \Omega, u$ and $\left\{b_{k}\right\}_{k \in \mathbb{N}}$ satisfy the hypotheses of the theorem. Fix $g_{0} \in \Omega$ and $r>0$ such that $B\left(g_{0}, 2 r\right) \subseteq \Omega$. For $k \in \mathbb{N}$, let $C_{k}=\left\{g \in \Omega \mid u(g)<b_{k}\right\}$. Then each $C_{k}$ is h-convex and measurable. Take $k \in \mathbb{N}$ large enough, in order to guarantee $u\left(g_{0}\right)<b_{k}$, whence $g_{0} \in C_{k}$, and suppose that $B\left(g_{0}, r\right) \backslash C_{k} \neq \emptyset$. By connectedness of $B\left(g_{0}, r\right)$, we can find $g \in B\left(g_{0}, r\right) \cap \partial C_{k}$. We have

$$
\frac{\mathcal{H}^{Q}\left(B(g, r) \backslash C_{k}\right)}{\mathcal{H}^{Q}(B(g, r))} \geq 1-c
$$

by Theorem 1.3 , where $0 \leq c<1$ does not depend on $k$. Hence,

$$
\frac{\mathcal{H}^{Q}\left(B\left(g_{0}, 2 r\right) \backslash C_{k}\right)}{\mathcal{H}^{Q}\left(B\left(g_{0}, 2 r\right)\right)} \geq \frac{1-c}{2^{Q}} .
$$

On the other hand, by hypothesis,

$$
\frac{\mathcal{H}^{Q}\left(B\left(g_{0}, 2 r\right) \backslash C_{k}\right)}{\mathcal{H}^{Q}\left(B\left(g_{0}, 2 r\right)\right)} \rightarrow 0 \quad \text { as } k \rightarrow+\infty .
$$


It follows that $B\left(g_{0}, r\right) \backslash C_{k}=\emptyset$ for large enough $k \in \mathbb{N}$. Thus, $u$ is locally bounded above in $\Omega$. The local Lipschitz continuity now follows from Lemma 3.1 and Proposition 3.2.

The author does not know whether there exist h-convex functions $u: \Omega \rightarrow \mathbb{R}$ which fail to satisfy the hypotheses of Theorem 6.5 .

\section{Acknowledgments}

It is my pleasure to thank Zoltán Balogh, Anders Björn, Roberto Monti, and Hans-Martin Reimann for their remarks and suggestions, which helped to improve both the content and presentation of the article.

\section{References}

[1] Ambrosio, L. and Magnani, V. Weak differentiability of BV functions on stratified groups, Math. Z. 245, 123-153, (2003).

[2] Balogh, Z. M. and Rickly, M. Regularity of convex functions on Heisenberg groups, Ann. Scuola Norm. Sup. Pisa Cl. Sci. (5) 2, 847-868, (2003).

[3] Björn, J. Boundary continuity for quasiminimizers on metric spaces, Illinois J. Math. 46, 383-403, (2002).

[4] Danielli, D. Regularity at the boundary for solutions of nonlinear subelliptic equations, Indiana Univ. Math. J. 44, 269-286, (1995).

[5] Danielli, D., Garofalo, N., and Nhieu, D.-M. Notions of convexity in Carnot groups. Comm. Anal. Geom. 11, 263-341, (2003).

[6] Danielli, D., Garofalo, N., Nhieu, D.-M., and Tournier, F. The Theorem of Busemann-Feller-Alexandrov in Carnot groups, Comm. Anal. Geom. 12, 853-886, (2004).

[7] Derridj, M. Sur un théorème de traces, Ann. Inst. Fourier (Grenoble) 22, 73-83, (1972).

[8] Evans, L. and Gariepy, R. Measure Theory and Fine Properties of Functions, Studies in Advanced Mathematics, CRC Press, Boca Raton, FL, (1992).

[9] Federer, H. Geometric Measure Theory, Reprint of the 1969 Edition, Classics in Mathematics, Springer-Verlag, Berlin, Heidelberg, (1996).

[10] Folland, G. B. and Stein, E. M. Hardy spaces on homogeneous groups, Math. Notes Princeton 28, Princeton University Press, NJ, (1982).

[11] Garofalo, N. and Tournier, F. New properties of convex functions in the Heisenberg group, Trans. Amer. Math. Soc. 358, 2011-2055, (2006).

[12] Gutiérrez, C. E. and Montanari, A. Maximum and comparison principles for convex functions on the Heisenberg group, Comm. Partial Differential Equations 29, 1305-1334, (2004).

[13] Gutiérrez, C. E. and Montanari, A. On the second order derivatives of convex functions on the Heisenberg group, Ann. Scuola Norm. Sup. Pisa Cl. Sci. (5) 3, 349-366, (2004).

[14] Lu, G., Manfredi, J., and Stroffolini, B. Convex functions on the Heisenberg group, Calc. Var. Partial Differential Equations 19, 1-22, (2004).

[15] Magnani, V. Lipschitz continuity, Aleksandrov Theorem and characterizations for H-convex functions, Math. Ann. 334, 199-233, (2006).

[16] Montefalcone, F. Some relations among volume, intrinsic perimeter and one-dimensional restrictions of BV functions in Carnot groups, Ann. Scuola Norm. Sup. Pisa Cl. Sci. (5) 4, 79-128, (2005).

[17] Monti, R. and Rickly, M. Geodetically convex sets in the Heisenberg group, J. Convex Anal. 12, 187-196, (2005).

[18] Rickly, M. On questions of existence and regularity related to notions of convexity in Carnot groups, PhD Thesis, University of Bern, (2005).

[19] Varadarajan, V.S. Lie Groups, Lie Algebras, and their Representations, Prentice-Hall Series in Modern Analysis, Prentice-Hall, Englewood Cliffs, NJ, (1974).

[20] Varopoulos, N. Th., Saloff-Coste, L., and Coulhon, T. Analysis and geometry on groups, Cambridge Tracts in Math. 100, Cambridge University Press, (1992).

[21] Wang, C. Viscosity convex functions on Carnot groups, Proc. Amer. Math. Soc. 133, 1247-1253, (2005). 
Received November 30, 2004

Institute of Mathematics, University of Bern, Sidlerstrasse 5, 3012 Bern, Switzerland e-mail: matthieu.rickly@math.unibe.ch

\author{
Communicated by Peter Li
}

